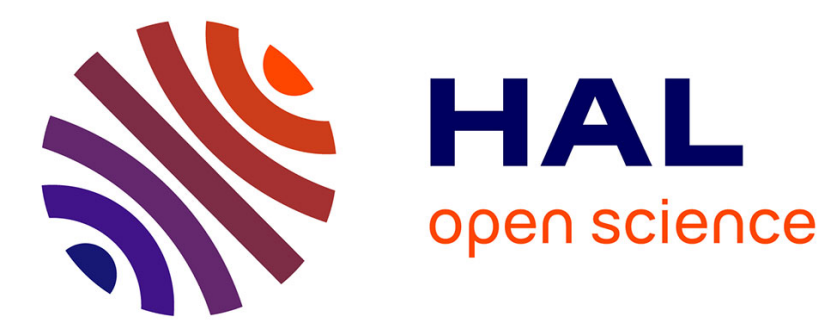

\title{
Electrostatics and Polarity in 2D Oxides
}

\author{
Claudine Noguera, Jacek Goniakowski
}

\section{To cite this version:}

Claudine Noguera, Jacek Goniakowski. Electrostatics and Polarity in 2D Oxides. Netzer, FP; Fortunelli, A. OXIDE MATERIALS AT THE TWO-DIMENSIONAL LIMIT, 234, SPRINGER-VERLAG BERLIN, pp.201-231, 2016, Springer Series in Materials Science, 978-3-319-28332-6; 978-3-319-28330-

2. 10.1007/978-3-319-28332-6_7 . hal-01403907

\section{HAL Id: hal-01403907 https://hal.science/hal-01403907}

Submitted on 7 Jul 2020

HAL is a multi-disciplinary open access archive for the deposit and dissemination of scientific research documents, whether they are published or not. The documents may come from teaching and research institutions in France or abroad, or from public or private research centers.
L'archive ouverte pluridisciplinaire HAL, est destinée au dépôt et à la diffusion de documents scientifiques de niveau recherche, publiés ou non, émanant des établissements d'enseignement et de recherche français ou étrangers, des laboratoires publics ou privés. 


\section{Contents}

Electrostatics and polarity in $2 \mathrm{D}$ oxides $\ldots \ldots \ldots \ldots \ldots \ldots \ldots \ldots \ldots$

Claudine Noguera and Jacek Goniakowski

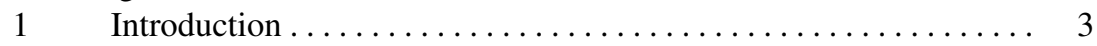

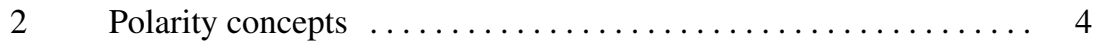

3 Polarity scenarios in thin films $\ldots \ldots \ldots \ldots \ldots \ldots \ldots \ldots \ldots \ldots \ldots$

3.1 Electronic reconstruction in polar films $\ldots \ldots \ldots \ldots \ldots \quad 8$

$3.2 \quad$ Uncompensated polarity ..................... 9

3.3 Structural transformation ..................... 10

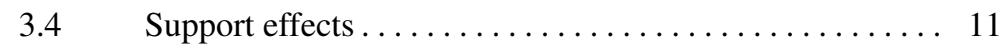

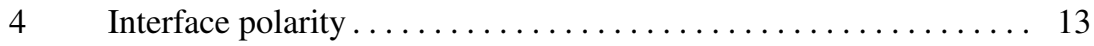

$4.1 \quad$ Criterion of compensation ................... 14

4.2 Electronic reconstruction at polar/polar interfaces...... 15

$4.3 \quad$ Finite size effects ........................... 16

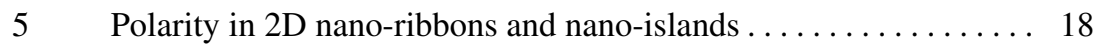

$5.1 \quad$ Electrostatic characteristics $\ldots \ldots \ldots \ldots \ldots \ldots \ldots \ldots \ldots$

$5.2 \quad$ Unsupported polar ribbons ..................... 19

$5.3 \quad$ Finite size effects....................... 22

$5.4 \quad$ Interfaces between polar ribbons . . . . . . . . . . . . . 23

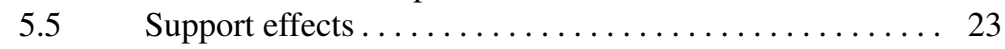

$5.6 \quad$ Polar nano-islands . . . . . . . . . . . . . . . . . . . . 25

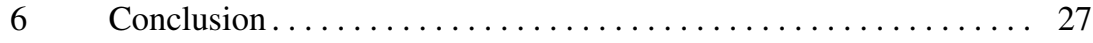

References ..................................... 27

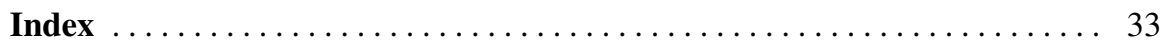





\title{
Electrostatics and polarity in 2D oxides
}

\author{
Claudine Noguera and Jacek Goniakowski
}

\begin{abstract}
We review the manifestations of electrostatic interactions, and in particular polarity effects, in oxide ultra-thin films and two-dimensional nano-objects. We discuss the efficiency of various compensation mechanisms such as overall structural transformations, strong lattice relaxations, inhomogeneous charge redistributions, support effects, and/or the formation of 2D or 1D electron/hole gases.
\end{abstract}

\section{Introduction}

Due to its relevance in mineralogy, geochemistry, catalysis, electrochemistry, electronics, magnetic recording, etc, in the past 25 years the field of oxide surfaces has enormously evolved. While the characteristics and properties of most binary oxide surfaces are now well-established, in the last decade, the main focus has been on ultra-thin oxide films. Usually grown on metallic supports, these systems may represent oxygen reservoirs, inverse catalysts, patterned supports for the formation of catalytically active size-selected clusters, or device constituents in the emerging field of all-oxide electronics. When the film thickness is no more than one or two atomic layers, depending upon preparation conditions, these two-dimensional (2D) oxides may display structures, stoichiometries and properties that are not seen in bulk crystals $[1,2,3,4,5,6]$. The same is expected in 2D small objects, such as

\section{Claudine Noguera}

CNRS, Institut des Nanosciences de Paris, UMR 7588, 4 place Jussieu, 75005 Paris, France Sorbonne Universités, UPMC Univ Paris 06, Institut des NanoSciences de Paris, F-75005, Paris, France

e-mail: claudine.noguera@insp.jussieu.fr

Jacek Goniakowski

CNRS, Institut des Nanosciences de Paris, UMR 7588, 4 place Jussieu, 75005 Paris, France

Sorbonne Universités, UPMC Univ Paris 06, Institut des NanoSciences de Paris, F-75005, Paris,

France

e-mail: jacek.goniakowski@insp.jussieu.fr 
nano-ribbons or nano-islands, whose controlled fabrication and growth nowadays remain a difficult challenge.

The present chapter focuses on the manifestations of electrostatic interactions in oxide ultra-thin films and 2D nano-objects, and in particular on polarity effects. Indeed, numerous oxide properties cannot be understood without taking into account the strong Coulomb interactions which result from their ionic character. Along certain crystallographic directions, a macroscopic polarization induces a surface instability. These polar orientations have been thoroughly studied at semi-infinite surfaces $[7,8]$. But in polar ultra-thin films, especially when their thickness reaches the monolayer (ML) limit, new questions arise, which are related to the role of electrostatic interactions and dimensionality. Compared to semi-infinite surfaces, new mechanisms of polarity compensation exist at the nanoscale, involving, among others, complete changes of structures, strong lattice relaxations, inhomogeneous charge redistributions. The interplay between polarity and substrate effects, interfacial charge transfers, and lattice mismatch, have also to be considered since most 2D-objects are grown on (metallic or insulating) substrates. Finally, the possibility to stabilize 2D or 1D electron or hole gases (2DEG, 2DHG, 1DEG, 1DHG) at polar interfaces or at polar island edges in new layered materials has recently generated excitement in the physics community.

The aim here is to present an overview of the consequences of the strong Coulomb interactions and polarity effects which exist in low dimensional oxide structures. We will outline how polarity concepts, which have been developed for surfaces and which are recalled in Section 2, apply or have to be modified at the nanoscale. For this purpose, they will be successively reviewed in the cases of ultrathin films (Section 3), interfaces (Section 4), and 2D ribbons or islands (Section $5)$.

\section{Polarity concepts}

In Tasker's classification [9], compound surfaces are differentiated according to whether or not the structural repeat unit starting from vacuum bears a non-vanishing dipole moment. The presence of a dipole moment is characteristic of polar surfaces.

In the simplest capacitor-type representation of a crystal cut along a polar orientation (Fig. 1), with alternating positively and negatively charged plates (charge densities $\pm \sigma$, successive inter-plate distances $R_{1}$ and $R_{2}$ ), an electrostatic instability - sometimes called polar catastrophe - develops due to the existence of a macroscopic electric field perpendicular to the surface. According to Gauss law, this instability can be healed by an excess charge density $\pm \delta \sigma$ on the external plates, such that:

$$
\delta \sigma=\sigma \mathscr{R}
$$


(a)

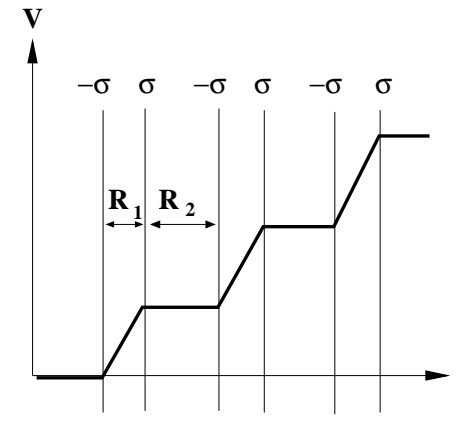

(b)

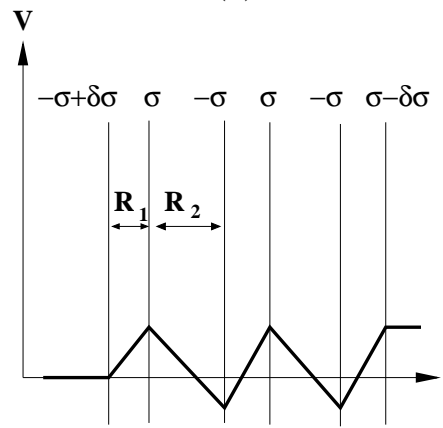

Fig. 1 (a): Capacitor model of a polar surface with alternating layers of charge densities $\pm \sigma$ and spatial variation of its electrostatic potential $V$. (b): Same but with excess charges $\pm \delta \sigma=$ $\sigma R_{1} /\left(R_{1}+R_{2}\right)$ on the outer layers which suppress the monotonic variation of $V$.

with $\mathscr{R}=R_{1} /\left(R_{1}+R_{2}\right)[7,8,9] . \sigma \mathscr{R}$ represents the dipole moment per unit volume of the repeat unit. The electrostatic field due to $\delta \sigma$ precisely cancels the average macroscopic electric field in the bulk of the material.

Beyond this simplistic picture, in realistic compounds, the charge density $\sigma$ of the atomic layers is delocalized and differs from the value $\Sigma$ obtained by assigning formal charges to the atoms. It involves an electronic contribution $\sigma_{e l}$ due to covalent effects, so that $\sigma=\Sigma+\sigma_{e l}$. Whatever the partitioning scheme adopted to define $\sigma$ and $\sigma_{e l}$, the electrostatic criterion, Eq. 1, defines the modification of surface charge density $\delta \sigma$ required for surface stability, which is then the sum of two contributions $\delta \sigma=\delta \sigma_{e l}+\delta \sigma_{\text {comp }}$. The first one, $\delta \sigma_{e l}$, comes from the modification of covalent effects at surfaces, due to the change in local environment of the atoms, associated, for example, to bond breaking. It exists whatever the surface orientation and is not specific to polarity. The second contribution, $\delta \sigma_{\text {comp }}$, represents the compensating charge which has to be provided by processes other than covalency effects, for example partial filling of surface bands, surface adsorbates, vacancies, non-stoichiometric reconstructions, or non-isoelectronic substitutions, in order to stabilize the surface. It is specific to polarity and, using Eq. 1, its value has to be equal to:

$$
\delta \sigma_{\text {comp }}=\Sigma \mathscr{R}+\left(\mathscr{R} \sigma_{e l}-\delta \sigma_{e l}\right)
$$

It consists of an ionic term $\Sigma \mathscr{R}$ plus an electronic one $\mathscr{R} \sigma_{e l}-\delta \sigma_{e l}$.

The Modern Theory of Polarization $[10,11,12]$ provides a formal derivation of this result. It relates the compensating charge, $\delta \sigma_{\text {comp }}$ to the bulk polarization $P_{b u l k}$ of the system, i.e. its dipole density, and writes the latter as:

$$
\delta \sigma_{\text {comp }}=P_{\text {bulk }} \quad ; \quad P_{\text {bulk }}=\Sigma \mathscr{R}+P_{e l}
$$

The electronic contribution $P_{e l}$, which depends on the center-of-gravity positions of the valence band (VB) Wannier functions, corresponds to the terms $\mathscr{R} \sigma_{e l}-\delta \sigma_{e l}$ 
written in Equation 2. $P_{e l}$ is non-zero in non-centro-symmetric structures in which the electronic density presents an asymmetry $[10,12,13,14]$.

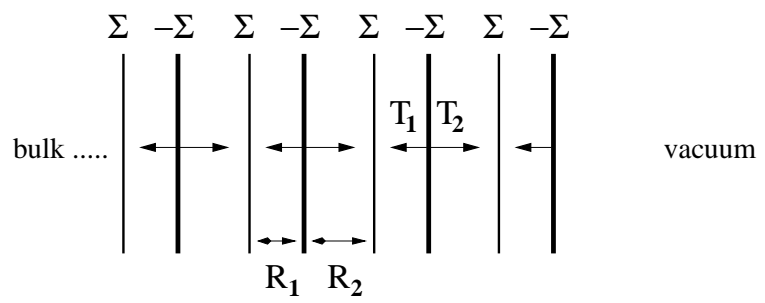

Fig. 2 Succession of layers along a direction perpendicular to a polar surface. $T_{1}$ and $T_{2}$ represent the interlayer electron transfers associated to $R_{1}$ and $R_{2}$ interlayer distances, respectively. $\pm \Sigma$ are the layer formal charge densities. Due to bond breaking, $T_{2}$ is missing at the surface.

Some qualitative insight into $P_{e l}$ is given by a simple tight-binding approach which accounts for electron delocalization through inter-layer transfers $T_{1}$ and $T_{2}$ per 2D unit cell ( $T_{1}$ and $\mathrm{T}_{2}$ positive), as shown in Fig. 2. In this particular partitioning of space, the electronic contribution to $\sigma$ is thus $\sigma_{e l}=-T_{1}-T_{2}$. At the surface $T_{2}$ is missing, which yields $\delta \sigma_{e l}=-T_{2}$. Local charge redistributions modifying the mixed ionic and covalent character of the bonding in the surface region, and thus $T_{1}$ and $T_{2}$, are irrelevant, since they induce no global charge variation. In this simple scheme, $\delta \sigma_{\text {comp }}$ and the electronic contribution to $P$ thus read:

$$
P_{e l}=\sigma_{e l}\left(\mathscr{R}-\frac{T_{2}}{T_{1}+T_{2}}\right)
$$

This expression, not intended to be used for quantitative evaluation, shows that symmetry considerations alone can tell whether or not $P_{e l}=0$. Indeed, the key issue for its cancellation is in the difference between $T_{2} /\left(T_{1}+T_{2}\right)$ and $\mathscr{R}$. It vanishes, for example, in rock salt (111) or zinc blende (100) compounds where layers are equidistant $\left(R_{1}=R_{2}\right.$ and $\left.\mathscr{R}=1 / 2\right)$ and $T_{1}=T_{2}$ by symmetry. Similarly, it vanishes in zinc blende (111) compounds where $R_{2}=3 R_{1}$ and, by symmetry $T_{1}=3 T_{2}$.

In wurtzite compounds, whether or not their structure is ideal, a spontaneous polarization of electronic origin exists, due to the absence of inversion symmetry in their lattice. Whenever $\Sigma \mathscr{R} \neq 0$, the electronic contribution $P_{e l}$ is often negligible, so that relying on a formal charge analysis most of the time allows a correct assignment of polarity. However, when $\Sigma \mathscr{R}=0$, either because $\Sigma=0$ or because $\Sigma \mathscr{R}$ is exactly canceled out by external charges, $P_{e l}$ remains the sole factor which drives polarity effects. An illustration of the case $\Sigma=0$ is given in Fig. 3, which displays the value of $P_{e l}$ and its consequences on surface polarity, in an hypothetical distorted $\left(R_{1} \neq R_{2}\right)$ perovskite bulk. In thin films or nano-objects, interfacial strain and global symmetry lowering may also induce non-vanishing and non-negligible $P_{e l}$, so that a formal charge analysis may not always be sufficient to assess the polar character of these nanoscale objects. 

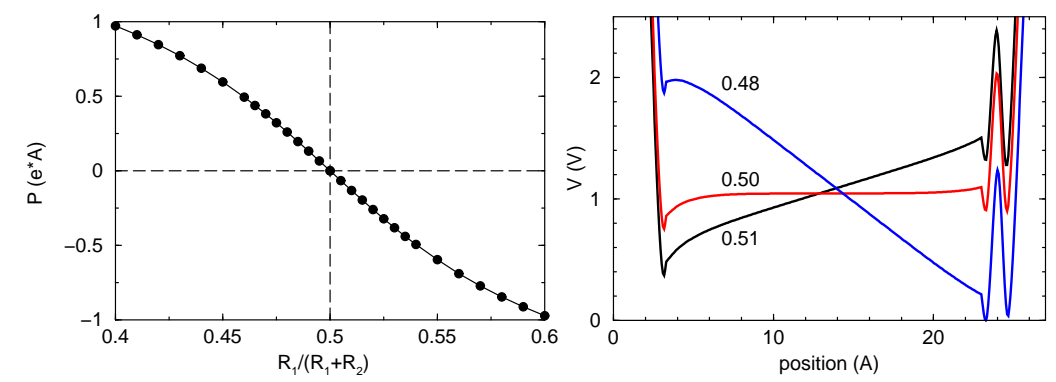

Fig. 3 Left: Polarization in an hypothetical distorted $\mathrm{SrTiO}_{3}$ bulk with flat, formally-neutral (001) layers, for various values of $\mathscr{R}$. Only when $\mathscr{R}=0.5$ does the polarization vanish. When $\mathscr{R} \neq 0.5$, since $\Sigma=0$, its non-zero value solely comes from its electronic contribution $P_{e l}$. Right: Electrostatic potential profile across a distorted $\mathrm{SrTiO}_{3}(001)$ slab for $\mathscr{R}=0.48,0.5$ and 0.51 . The non-zero slope when $\mathscr{R} \neq 0.5$ is the signature of polarity.

One can remark that polar semi-infinite surfaces are characterized by a polarization discontinuity $\delta P$ between a polar compound $\left(P_{b u l k} \neq 0\right)$ and vacuum, which is a medium with zero polarization $(P=0)$. The same is true for polar surfaces in contact with any non-polar medium [15], to which all previous considerations apply.

In order to provide the compensating charge densities $\delta \sigma_{\text {comp }}=P_{\text {bulk }}$ required for surface stabilization, several mechanisms may be at work, whose relative efficiency is strongly dependent on the oxide, the surface atomic structure, the chemical environment or the thermodynamic conditions (temperature, oxygen or water partial pressures). As reviewed in Refs. [7, 8], a modification of the surface region composition by an adequate density of charged vacancies, or the adsorption of an adequate density of charged foreign species, in particular protons or hydroxyl groups resulting from the dissociation of water molecules, are the most commonly met. Whenever $P_{e l} \neq 0$, it should be kept in mind that these required densities are close to, but not equal to $\mathscr{R}$. This is for example the case at wurtzite (0001) surfaces, whether ideal or not. There are also examples of surface configurations consistent with a metallization mechanism, sometimes called electronic reconstruction, in which a modification of the surface band filling (Zener breakdown) induces the presence of $2 \mathrm{DEG} / 2 \mathrm{DHG}$ at the surface.

\section{Polarity scenarios in thin films}

As reviewed in Refs. $[8,16]$, the physics of polar thin films is very rich in new electronic and structural features, due to finite size effects, structural flexibility, softening of the electrostatic interactions and interaction with the substrate. In particular, at variance with semi-infinite surfaces, the stability of polar film does not necessar- 
ily require $\delta \sigma_{\text {comp }}=P_{\text {bulk }}$. We will successively describe several scenarios that may take place.

\subsection{Electronic reconstruction in polar films}

As evidenced for example in $\mathrm{MgO}(111)$ [17, 18], $\mathrm{CoO}(111)$ [19] or $\mathrm{ZnO}(0001)$ $[20,21]$ films, two qualitatively different thickness regimes may be differentiated. At large thicknesses, the polarity stabilization mechanisms are qualitatively similar to those occurring at semi-infinite surfaces. However, the electronic reconstruction scenario displays finite size characteristics, that are specified below.

(a)

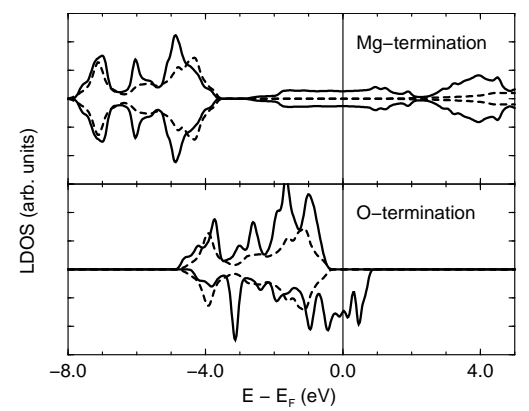

(b)

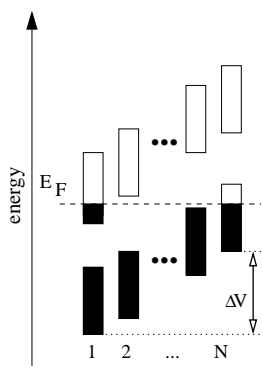

(c)

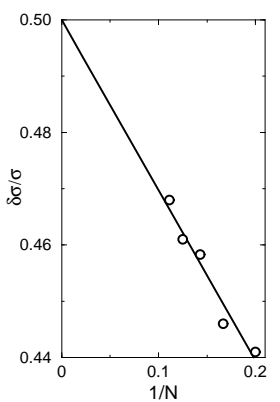

Fig. 4 (a): Spin resolved LDOS on the magnesium (top) and oxygen (bottom) terminations of a $\mathrm{MgO}(111)$ film, highlighting the metallization of both surfaces and the magnetic moment on the oxygen termination. (b): Sketch of the electronic structure across a polar thick film. (c) Thickness dependence of $\delta \sigma / \sigma$, deduced from a Bader analysis, in thick $\mathrm{MgO}(111)$ films, evidencing its $1 / N$ asymptotic behavior.

Simulations of the electronic structure of thick polar films evidence metallization and spin polarization at their surfaces (Figure 4a). The local VB on one side of the film and the local conduction band (CB) on the other side intersect the Fermi level. This allows a reduction of charge density $\pm \delta \sigma$ on both terminations, through electron exchange between them (Zener breakdown). More precisely, the local densities of states (LDOS) are monotonically shifted across the film, due to a residual polarization coming from the opposing effect of the film polarization $P$ and $\delta \sigma$ (Figure $4 \mathrm{~b})$. The associated total band shift $\Delta V$, no longer proportional to the film thickness, remains of the order of the surface gap $(G)$ to allow band overlap and electron transfer.

The self-consistent relationship between $\Delta V$ and $\delta \sigma$ constrains the latter to vary with the film thickness $N\left(R_{1}+R_{2}\right)$ ( $N$ the number of repeat units), according to the asymptotic law $[22,23]$ ( $\varepsilon^{\infty}$ the film dielectric constant): 


$$
\delta \sigma_{\text {comp }}(N) \approx P_{\text {bulk }}-\frac{\Delta V \varepsilon^{\infty}}{4 \pi N\left(R_{1}+R_{2}\right)}
$$

An example of the $1 / N$ dependence of $\delta \sigma$, found in $\mathrm{MgO}(111)$ films, is shown in Fig. 4c. Thus, in these finite size systems, $\delta \sigma_{\text {comp }}$ differs from the bulk polarization $P_{\text {bulk }}$ :

$$
\delta \sigma_{\text {comp }}(N) \neq P_{\text {bulk }}
$$

whenever $G$ is non-zero. Only in the $N \rightarrow \infty$ limit is the equality recovered.

\subsection{Uncompensated polarity}

Under specific conditions, the total shift of electrostatic potential $\Delta V$ may be insufficient to induce band overlap. This happens, either at extremely low thickness without much structural distortion $\left(P \approx P_{b u l k}\right)$, or at low thickness if the film structure is flexible enough to strongly reduce its polarization with respect to the bulk value $\left(P \ll P_{\text {bulk }}\right)$. Whatever the case, $\delta \sigma_{\text {comp }} \neq P$ since

$$
\delta \sigma_{\text {comp }}=0
$$

For this reason, this regime has been called "uncompensated polarity" [18]. In another context, it is referred to as the subcritical regime [24]. The associated electronic structure is sketched in Fig. 5a.

This scenario was discovered in a theoretical study of stoichiometric $\mathrm{MgO}(111)$, $\mathrm{ZnO}(0001)$ and $\mathrm{NaCl}(111)$ ultra-thin films, which display a metastable zinc blende phase at low thickness with strongly size-dependent properties [18]. As shown in Fig. 5 b, $\mathscr{R}$ is extremely reduced thanks to a drastic decrease of the inter-layer distance $R_{1}$. The total dipole moment grows linearly with $N$ and simultaneously, $G$ decreases. The electronic structure remains insulating but only up to a critical thickness $N_{c}$. At $N_{c}$, a metal-insulator transition takes place with a strong discontinuity in $\mathscr{R}$ and $\delta \sigma$. Above $N_{c}$, all film properties follow the general trends of compensation by metallization described in the previous section

Kaolinite films have also been predicted to display the same scenario, although the uncompensated mechanism was not referred to [25]. Kaolinite $\mathrm{Al}_{2} \mathrm{Si}_{2} \mathrm{O}_{5}(\mathrm{OH})_{4}$ is a layered silicate compound, whose (0001) layers are composed of two sublattices, made of a silica and a gibbsite sheet, respectively. Due to this asymmetry, the repeat unit along the (0001) orientation bears a finite dipole moment, making this orientation polar. According to the simulation, kaolinite (0001) films display a metal insulator transition when the thickness reaches about three repeat units.

A nearly identical scenario has been invoked in $\mathrm{LaAlO}_{3}(100)$ and $\mathrm{KTaO}_{3}(100)$ films grown on $\mathrm{SrTiO}_{3}$ substrate to rationalize experimental results[26, 27, 28, 29, $30]$. It gave rise to an intense debate on whether the $2 D E G$ originates from polarity or/and from the presence of oxygen vacancies [31, 32]. Indeed, these oxides are insulating, but the $\mathrm{LaAlO}_{3}(100)$ and $\mathrm{KTaO}_{3}(100)$ orientations are polar. For example, 
(a)

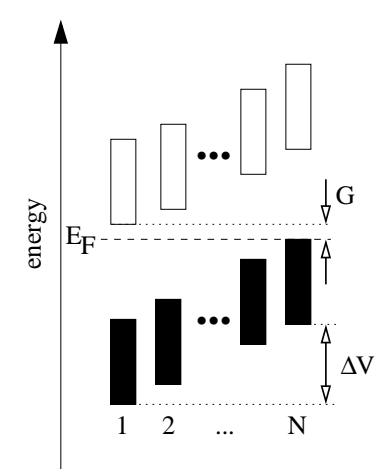

(b)

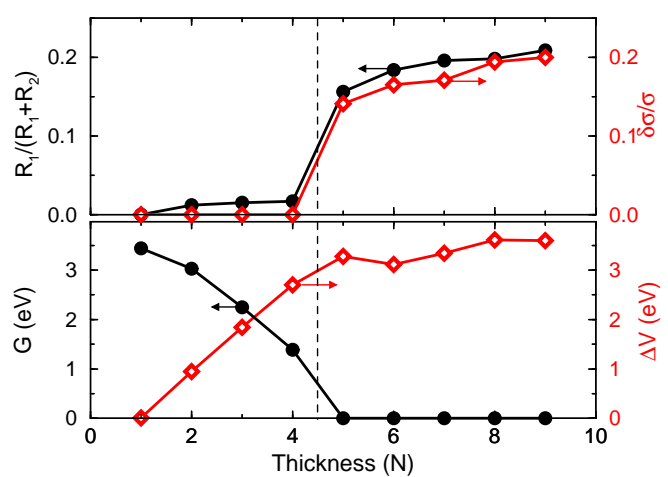

Fig. 5 (a): Sketch of the electronic structure across a polar uncompensated film for $N<N_{C}$; (b) thickness dependence of unsupported $\mathrm{MgO}$ (111) film properties in the metastable zinc blende phase. Top panel: $\mathscr{R}=R_{1} /\left(R_{1}+R_{2}\right)$ (black symbols) and excess Bader charge densities $\delta \sigma / \sigma$ (red open symbols); Bottom panel: gap $G$ (black symbols) and total voltage $\Delta V$ (red open symbols).

in $\mathrm{LaAlO}_{3}$, a metal-insulator transition occurs at a critical thickness of four repeat units $[24,26]$. This transition was assigned to the shift of the $\mathrm{LaAlO}_{3}$ bands, which, beyond $N_{c}$, allows its top of $\mathrm{VB}$ to overlap the $\mathrm{SrTiO}_{3} \mathrm{CB}$ minimum.

\subsection{Structural transformation}

The literature review on polar ultra-thin films $[8,16]$ evidences another scenario at low thickness, in which a global structural transformation takes place in order to make the film orientation non-polar. In that case, the equality $\delta \sigma_{\text {comp }}=P$ is fulfilled, but in the specific limit where:

$$
P=0=\delta \sigma_{\text {comp }}
$$

Such transformation results from a competition between surface and bulk energy terms, in which the surface term wins. The new structure being different from the bulk ground state, there is a cost of energy for each additional repeat unit when the thickness $N$ increases. However, as long as $N$ remains small, the gain in surface energy in having a non-polar orientation is able to overcome this cost. Thus, up to a critical thickness, ultra-thin polar films may sustain a structure which is not their bulk ground state but allows a non-polar termination.

This generic scenario was first proposed in Ref. [17], accompanying simulations of $\mathrm{MgO}(111)$ films (Figure 6a). It rationalizes theoretical or experimental findings made in many polar binary oxide films, such as $\mathrm{MgO}$ [33], $\mathrm{FeO}[34,35], \mathrm{CoO}$ $[36,37], \mathrm{ZnO}[38,39]$, BeO [40, 41], other wurtzite type compounds [42], and 
$\mathrm{AlO}_{x}$ [43], which evidence nearly flat repeat units and expanded in-plane lattice parameters compared to bulk. Among the reported results, a frequently-met feature is the existence of flat layers with a graphene-like honeycomb structure made of six-member rings with equal number of cations and oxygens (Figure 6b). When such layers are stacked together, a non-polar (0001) film of an hexagonal boron nitride structure (h-BN) is produced. This scenario is relevant for binary compounds which, in the bulk, crystallize in the rock salt, zinc blende or wurtzite structures. More generally, the stabilization of polar films by a strong transformation of the bulk equilibrium structure appears to be a quite general scenario at extremely low thickness, due to the structural flexibility of ultra-thin films.
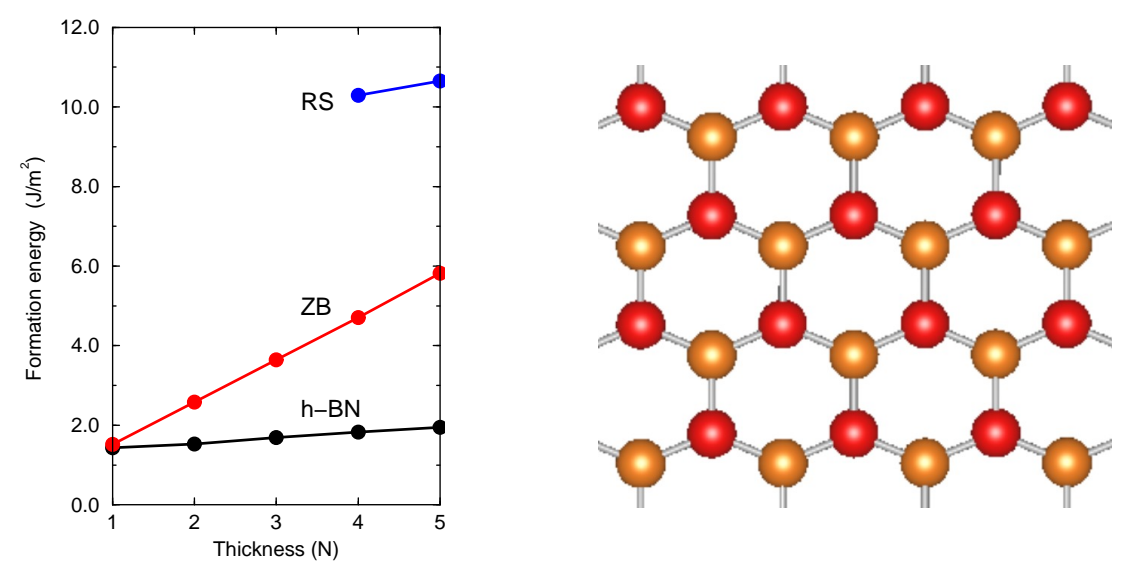

Fig. 6 (a): Structural phase diagram of $\mathrm{MgO}(111)$ unsupported films as a function of thickness, indicating the relative stability of the rock salt (RS), zinc blende (ZB) and h-BN phases. (b): Top view of the honeycomb structure of the h-BN ML.

\subsection{Support effects}

While previous scenarios are intrinsic to (unsupported) polar thin films, the interaction with a support can significantly modify the film structure, their stability and their electronic characteristics, depending on their thickness.

At the interface between a thick polar film and a metal substrate, the cost of polarity is strongly reduced by a transfer of the compensating charge from the oxide to the metal $[44,45]$. The interfacial oxide bands recover their normal filling, and it is the filling of the metal bands in contact with the oxide which changes with respect to that of the clean metal surface. This transfer avoids electron excitations across the oxide gap and replaces them by low-energy excitations in the vicinity of the metal Fermi level. The associated gain of energy is reflected in a strong interfa- 
cial adhesion. This process was invoked to support the experimental evidence of a structurally and chemically abrupt $\mathrm{Cu} / \mathrm{MgO}(111)$ interface $[46,47]$.

When the oxide film is thin and especially when polarity effects are weak or absent (case of uncompensated polarity or structural transformation), an interfacial charge transfer of a different origin takes place between the film and the metal substrate, which is no longer driven by the requirement of polarity compensation. Due to interfacial hybridization and/or penetration of the MIGS (Metal Induced Gap States), its amount and sign depend on the relative position of the metal Fermi level and the oxide point of zero charge. The associated dipole moment contributes to the Schottky barrier height [48, 49, 50]. For example, deposition of a $\mathrm{MgO}(111)$ film on a simple metal $(\mathrm{Mg}, \mathrm{Al})$ of low electronegativity results in an electron transfer from the substrate to the film, while on transition metal substrates (Ag, Mo, Pt) an opposite transfer occurs. Such charge transfer is unrelated to polarity as it may also occur at the interface between a metal and a non-polar surface.

(a)

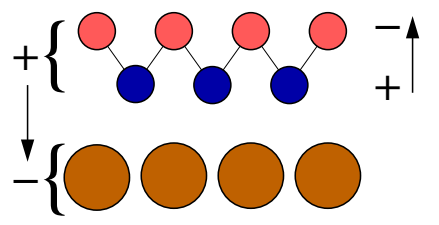

(b)

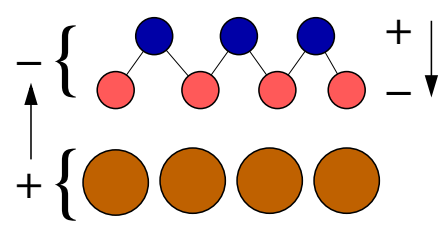

Fig. 7 Schematic representation of the two dipoles associated to the charge transfer between an oxide film and its metal support and to the rumpling inside the oxide film (shown by arrows), for the two cases of a negative (a) and a positive (b) metal charging. In the first case, oxygen atoms of the oxide film are repelled by the negative charge of the metal and pushed outwards. In the second case, they are attracted by the substrate. Cations, oxygens, and metal atoms are represented as blue, red and large brown balls, respectively.

As a response to the electrostatic field produced by the interfacial charge transfer, thin films undergo a structural distortion as schematized in Fig. 7 in the case of an oxide ML of honeycomb structure deposited on a metal substrate. While perfectly flat when unsupported, the ML gets rumpled upon deposition, in such a way that the dipoles associated to charge transfer and rumpling have opposite sign and thus partially compensate each other [51]. This argument based on a generic electrostatic coupling between charge transfer and structural distortion rationalizes the small and large rumpling values in $\mathrm{MgO} / \mathrm{Ag}(111)$ and $\mathrm{FeO} / \mathrm{Pt}(111) \mathrm{ML}$ films, respectively.

A similar electrostatic coupling between charge transfer and structural distortion applies to adsorbates on thin oxide films supported on a metal support. Upon deposition, the adsorbates become charged, in a way which is mainly determined by the electronic characteristics (e.g. the work function) of the oxide-on-metal support $[52,53,54,55,56,57,58,59,60,61,62]$. In response to this charge transfer, especially at low film thickness, a polaronic distortion of the oxide film takes place around the charged adsorbates, which significantly stabilizes them [63] and has im- 
plications on the growth, chemical, optical, and magnetic properties of adsorbed metal particles, their self-organization $[3,64]$ and their wetting on the oxide film $[54,58]$.

It has furthermore been demonstrated that this mechanism allows to incorporate oxygen atoms into ultra-thin oxide films supported on a metal, producing oxygenrich oxide phases, with no bulk equivalent, which may serve as oxygen reservoirs in low temperature oxidation reactions $[65,66]$. A prototypical example is the $\mathrm{FeO}$ ML on $\mathrm{Pt}(111)$ which, upon interaction with an oxygen atmosphere, transforms into $\mathrm{O}-\mathrm{Fe}-\mathrm{O}$ trilayer islands stabilized by a strong interfacial charge transfer [67, 68]. A similar mechanism is also likely active for other O-M-O trilayers, such as $\mathrm{IrO}_{2}$ [69], $\mathrm{PdO}_{2}$ [70], $\mathrm{RhO}_{2}$ [71], $\mathrm{RuO}_{2}$ [72] and $\mathrm{MnO}_{2}$ [73, 74].

\section{Interface polarity}

Oxide/oxide interfaces have recently focused the attention of the research community, due to the entirely new properties they display compared to their parent materials, and to their much wider variety of behaviors compared to traditional semiconductor/semiconductor interfaces $[75,76]$. The possibility of producing a confined 2DEG/2DHG, subject of enhanced correlation effects, and magnetic or even superconducting instabilities, represents a particularly exciting issue. This section focuses on the compensation scenarios which may take place at polar/polar interfaces. We will not consider polar/non-polar interfaces for which, as stated in Section 2, the absence of polarization in one of the compounds makes their polarity characteristics similar to those described at the interface between polar films and vacuum.
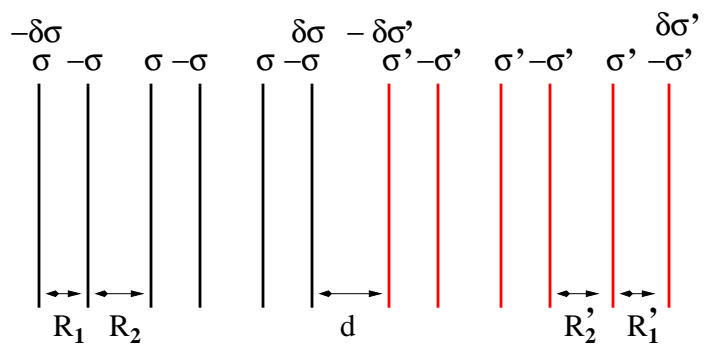

Fig. 8 Capacitor model of a polar/polar interface, assuming contact between two layers of opposite character. In an heterostructure, the two outer layers (extreme left and right layers) are free surfaces in contact with vacuum. In superlattices, they are in contact with each other, via the application of periodic boundary conditions. In that case, the outer interface charge density is equal and opposite to that in the central interface. 


\subsection{Criterion of compensation}

A straightforward extension of Equations 1 and 3 to polar/polar interfaces in the large thickness limit (Figure 8), yields the following electrostatic criterion for the stability of the interface:

$$
\delta \sigma=\mathscr{R} \sigma \quad ; \quad \delta \sigma^{\prime}=\mathscr{R}^{\prime} \sigma^{\prime}
$$

The excess charge densities $\delta \sigma$ and $\delta \sigma^{\prime}$ are required at the polar terminations of each medium, leading to an excess interface charge density:

$$
\delta \sigma^{I}=\delta \sigma-\delta \sigma^{\prime}
$$

Aside from those provided by covalent contributions, the remaining compensating charges leading to the cancellation of the polarizations $P$ and $P^{\prime}$ present in the core of each subsystem read:

$$
\delta \sigma_{\text {comp }}=P=\mathscr{R} \Sigma+P_{e l} \quad ; \quad \delta \sigma_{\text {comp }}^{\prime}=P^{\prime}=\mathscr{R}^{\prime} \Sigma^{\prime}+P_{\text {el }}^{\prime}
$$

The interfacial compensating charge densities $\pm \delta \sigma_{\text {comp }}^{I}$ are equal to the polarization discontinuity $\delta P=P-P^{\prime}$ between the two media:

$$
\delta \sigma_{\text {comp }}^{I}=\delta \sigma_{\text {comp }}-\delta \sigma_{\text {comp }}^{\prime}=\delta P
$$

At a polar/polar interface, both polarizations $P$ and $P^{\prime}$ are different from zero. Using Equation 11, the polarization discontinuity can be written under the following form [77]:

$$
\delta P=P-P^{\prime}=\mathscr{R}\left(\Sigma-\Sigma^{\prime}\right)+\Sigma^{\prime}\left(\mathscr{R}-\mathscr{R}^{\prime}\right)+\delta P_{e l}
$$

which highlights its three contributions:

- the term $\mathscr{R}\left(\Sigma-\Sigma^{\prime}\right)$ depends on the difference of formal charge densities $\Sigma-\Sigma^{\prime}$ between the two parts of the system. It represents the contribution from valence discontinuity.

- the term $\Sigma^{\prime}\left(\mathscr{R}-\mathscr{R}^{\prime}\right)$ represents the contribution from structural discontinuity.

- the term $\delta P_{e l}$ depends on the difference of the electronic contributions to $P$ and $P^{\prime}$. It thus represents the electronic discontinuity. It is non-zero when one or both compounds are non-centro-symmetric.

Polarity compensation mechanisms at polar/polar interfaces may be extrapolated from those already mentioned at polar surfaces. They may involve interface nonstoichiometry (including charged vacancies or interfacial mixing [78]), structural reconstructions [79] or electronic reconstruction. In the field of oxide/oxide interfaces, the latter mechanism has been experimentally evidenced at the polar/polar $\mathrm{ZnO}(0001) / \mathrm{Zn}_{x} \mathrm{Mg}_{1-x} \mathrm{O}(0001)$ interface [80]. 


\subsection{Electronic reconstruction at polar/polar interfaces}

In this section, we specify the conditions under which a 2DEG may exist at the interface between two stoichiometric polar compounds [77].
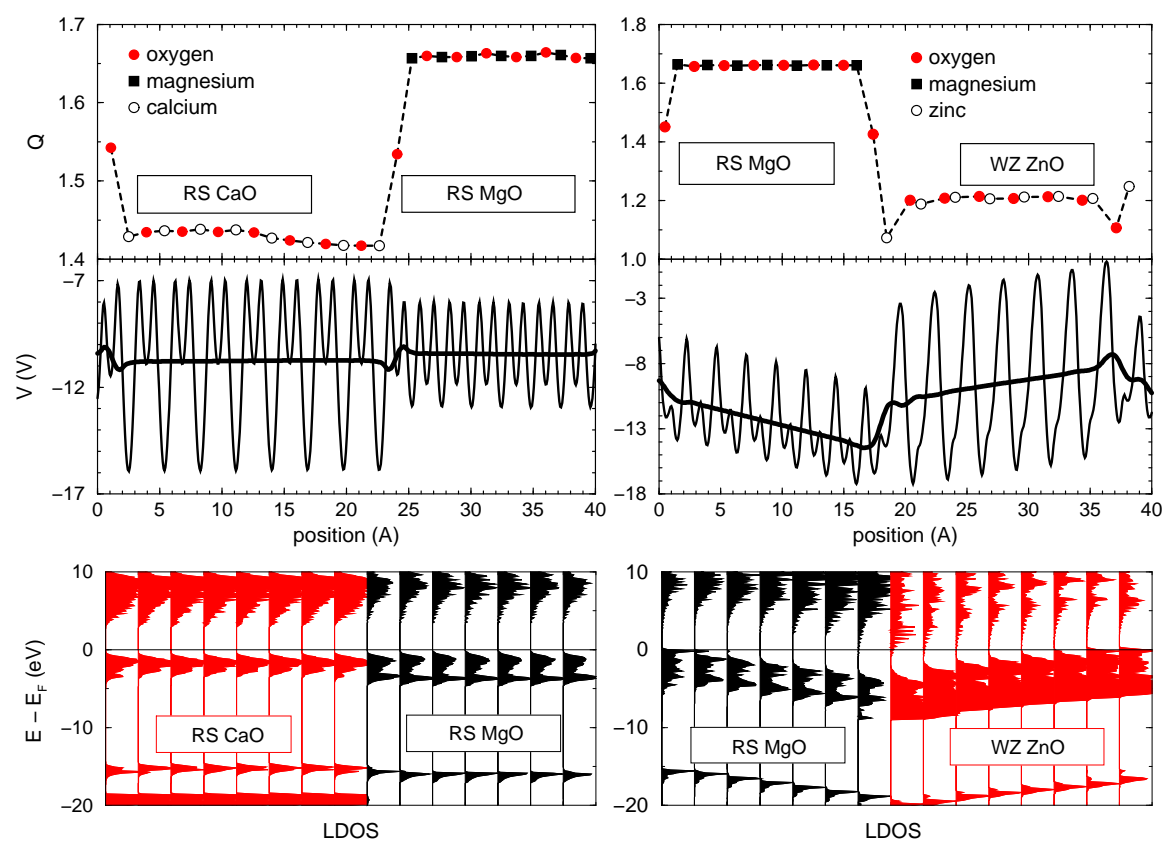

Fig. 9 Electronic properties of rock salt $\mathrm{CaO}(111) /$ rock salt $\mathrm{MgO}(111)$ (left panels) and rock salt $\mathrm{MgO}(111) /$ wurtzite $\mathrm{ZnO}(0001)$ (right panels) superlattices. From top to bottom: Bader charges $Q$ (absolute values), electrostatic potential profiles $V$, and LDOS. The thin line in the potential profiles represent its lateral average $\bar{V}$, while the thick one corresponds to its cell average $\overline{\bar{V}}$.

A first situation arises when $\delta P=0$. Although, in that case, $\delta \sigma_{\text {comp }}^{I}=0$, nonnegligible charge modifications may take place at the interface, which reveal the electronic contribution to $\delta \sigma^{I}$. There is no need of electronic or structural reconstruction at the interface, which remains insulating. The profile of the electrostatic potential across the system is flat and there is no residual electric field on both sides of the interface. The interface is thus non polar, although each sub-system on its own is polar. For this condition to be fulfilled, there needs to be no structural discontinuity, no valence discontinuity and no electronic contribution to polarization on both sides of the interface. $\delta P=0$ occurs at ideal zinc blende/zinc blende interfaces when the two compounds have the same valency, at ideal rock salt/rock salt (RS) interfaces (as exemplified in Fig. 9 in the particular case of $\mathrm{MgO}(111) / \mathrm{CaO}(111)$ interface [77]), at ideal perovskite/perovskite interface such as $\mathrm{KSbO}_{3}(001) / \mathrm{KNbO}_{3}(001)$ 
[81]. The $\mathrm{SrTiO}_{3}(110) / \mathrm{LaAlO}_{3}(110)$ interface studied in Refs. [82, 83] between two perovskites aligned along the polar (110) direction represents a special case. If stoichiometric, this interface is non-polar, since no polarization discontinuity exists between the two materials. However, in the experiment, a metal-insulator transition was evidenced and assigned to polarity effects induced by an interfacial mixing.

A second type of polar/polar interfaces is characterized by $\delta P \neq 0$, when one or several of its three contributions (structural, valence, or electronic discontinuity) are non zero. Such interfaces are polar, with a strong voltage across the system, which has to be canceled out, either by interface non-stoichiometry, as in the $\mathrm{ZnO} / \mathrm{FeO}$ interface [84], or by the formation of a 2DEG, depending upon experimental preparation conditions. For the latter to exist, an overlap between the top of the VB of one compound and the bottom of the $\mathrm{CB}$ of the other compound has to take place, as illustrated in Fig. 9 in the case of wurtzite (WZ) $\mathrm{ZnO}(0001) /$ rock salt $\mathrm{MgO}(111)$ interface [77] and recently recognized in Ref. [15]. The $\mathrm{ZnO}(0001) / \mathrm{Zn}_{x} \mathrm{Mg}_{1-x} \mathrm{O}(0001)$ interface, in which Shubnikov-de Haas oscillations and the quantum Hall effect have been observed [80], also belongs to the family of polar compensated interfaces.

\subsection{Finite size effects}

Similarly to what occurs in polar ultra-thin films, polar/polar heterostructures or superlattices involving compounds of finite thickness may display properties modified by finite size effects. The latter may lead to the existence of a subcritical regime or to a structural transformation to avoid polarity.

An uncompensated polarity regime may take place at low thickness, either without much structural distortion (case of a small polarization discontinuity between the two bulk compounds $\delta P_{\text {bulk }}$ ), or when the film structure is flexible enough to strongly reduce the polarization discontinuity $\left(\delta P \ll \delta P_{b u l k}\right)$. Under these circumstances, the voltage $\Delta V$ due to $\delta P$ is too small to induce an overlap of the VB of one compound with the $\mathrm{CB}$ of the other one at the interface and $\delta \sigma_{\text {comp }}^{I}=0$. Eq. 12 is thus not fulfilled in this regime $\left(\delta \sigma_{\text {comp }}^{I} \neq \delta P\right)$. The interface remains insulating up to a critical thickness $d_{c}$ of the order of $\Delta V / \delta \sigma_{\text {comp }}^{I}$ and displays a metal-insulator transition at $d_{c}[77]$.

If $\delta P$ results from a valence discontinuity or a strong structural discontinuity, the critical thickness will be extremely small and the pre-critical regime will likely not be observable. If, however, the polarization discontinuity and thus $\delta \sigma_{\text {comp }}^{I}$ are small enough, $d_{c}$ may involve up to several tens of repeat units. This may happen at the interface between e.g. two wurtzite compounds with $\Sigma=\Sigma^{\prime}$. As an example, Fig. 10 displays the interfacial characteristics of an $\mathrm{AlN}(0001) / \mathrm{GaN}(0001)$ superlattice [77]. Both compounds crystallize in a non-ideal wurzite structures with slightly different $\mathscr{R}$. $\delta P$ thus results from a weak structural discontinuity and from an electronic contribution $\delta P_{e l}$ due to the non-centro-symmetric wurtzite structure. The average potential $\overline{\bar{V}}$ profile presents a small slope, which yields small band shifts. Below a 

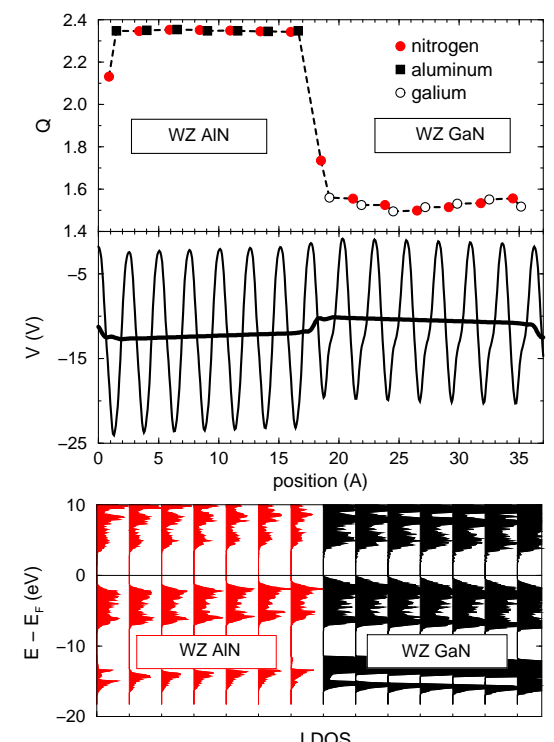

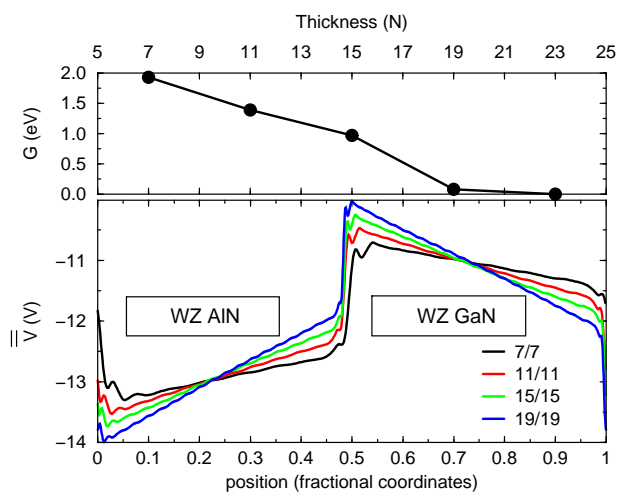

Fig. 10 Left panels: same as in Fig. 9 for a wurtzite $\mathrm{AlN}(0001) /$ wurtzite $\mathrm{GaN}(0001)$ superlattice. At the thickness under consideration, the interfaces are insulating. Right panels, from top to bottom: Thickness dependence of the gap width $G$ and cell-averaged electrostatic potential profiles (in fractional coordinates), in superlattices of increasing thicknesses.

critical thickness of about twenty repeat units, $\Delta \overline{\bar{V}}$ is insufficient to induce a band overlap and the interfaces remain insulating. A similar behavior has been found in a simulation of the polar/polar $\mathrm{ZnO}(0001) / \mathrm{MgO}(0001)$ interface at low thickness, assuming wurtzite structure for $\mathrm{MgO}$ [85]. Above $d_{c}$, $\mathrm{VB}$ and $\mathrm{CB}$ overlap, which provides the compensating interfacial charges, and an interfacial 2DEG occurs, as found experimentally in $\mathrm{ZnO}(0001) / \mathrm{ZnMgO}(0001)$ superlattices in the regime of low Mg doping [80, 86].

Aside from the existence of a subcritical regime, other finite size effects may occur. At small thickness, atomic layers are flexible and may depart from the bulk geometry, either intrinsically or in order to accommodate the interfacial strain. As described in the thin film section, a layer flattening towards a honeycomb structure may allow to avoid polarity at low thickness in thin rock salt or wurtzite films.

These remarks stress that, in superlattices or heterostructures made of thin layers, predicting polarity character from the bulk properties of the two materials may lead to erroneous results. In the case of interfaces with large polarization discontinuity, finite size effects are not likely to alter the polar character of the interface, except if a structural transformation towards non-polar orientations takes place. In other systems, they may modify the structural and the electronic contributions to the polarization discontinuity, and thus the critical thickness of the metal-insulator transition. They may also induce a finite polarization discontinuity in systems in which bulk considerations predict $\delta P=0$. 


\section{Polarity in 2D nano-ribbons and nano-islands}

While polarity concepts in ultra-thin films are rather well-established, the same is not true in 2D nano-ribbons and nano-islands [16]. A surge of interest in this field has taken place after the discovery of edge states at the Fermi level of graphene zigzag (ZZ) nano-ribbons [87, 88], although, as it will appear clearly, their physical origin is different. It is not often well-recognized that, depending upon the orientation of their edges, 2D objects may have a non-vanishing in-plane polarization. Experimentally, the difficulty in producing them in a well-characterized and reproducible way explains why most information that we have come from atomistic simulations.

\subsection{Electrostatic characteristics}

Nano-ribbons with infinite edges are the 2D equivalents of thin films. When their 2D repeat unit bears a dipole moment perpendicular to their edges, the latter are polar. The polarity characteristics then depend on the charge density per unit length of the atomic rows $\pm \lambda$, the geometric factor associated to inter-row distances $\mathscr{R}=$ $R_{1} /\left(R_{1}+R_{2}\right)$ and the ribbon width $H=N\left(R_{1}+R_{2}\right)$ ( $N$ the number of repeat units perpendicular to the edge).

Nano-ribbons may be produced from (001) ML of rock salt structure. Edges along the [110] direction $(\mathscr{R}=1 / 2)$ are polar, while those along [100] directions are non-polar. Alternatively, starting from the honeycomb-like (0001) ML of the h$\mathrm{BN}$ structure, ribbons with $\mathrm{ZZ}$ or armchair (AC) edges can be obtained. The former are $\operatorname{polar}(\mathscr{R}=1 / 3)$ and the latter non-polar. Ribbons cut out of a $1 \mathrm{H}(0001)$ trilayer of transition metal dichalcogenides (TMD) may also have $\mathrm{ZZ}$ or AC edges. Figures. $11 \mathrm{a}$ and $\mathrm{b}$ display top views of polar [110] and ZZ ribbons.

The main electrostatic difference between polar thin films and polar ribbons lies in the logarithmic variation of the electrostatic potential $V(z)$ across a ribbon and the logarithmic dependence of the potential jump $\Delta V$ as a function of its width $H$ (Figure $11 \mathrm{c}$ ) [89]. Contrary to thin films in which $\Delta V$ and the total dipole moment scale linearly with $N$, in polar ribbons in the absence of compensating charges, $\Delta V$ grows as $\ln N$ while the total dipole moment diverges linearly with $N$. Such logarithmic asymptotic behavior of $\Delta V$ and the absence of proportionality between $\Delta V$ and the total dipole moment are characteristic of electrostatic fields in 2D.

In the large width limit, the stability of polar ribbons requires edge excess charge densities $\delta \lambda$ (per unit length), whose expression is formally similar to that for $\delta \sigma$ in polar films:

$$
\delta \lambda=\mathscr{R} \lambda
$$

and the expression of the compensating charges $\delta \lambda_{\text {comp }}$, as a function of the $2 \mathrm{D}$ polarization $P_{M L}$ (now referred to the full ML) and the formal charge densities $\Lambda$ borne by the rows, reads: 


$$
\delta \lambda_{\text {comp }}=P_{M L}=\Lambda \mathscr{R}+P_{e l}
$$

(b)

(c)

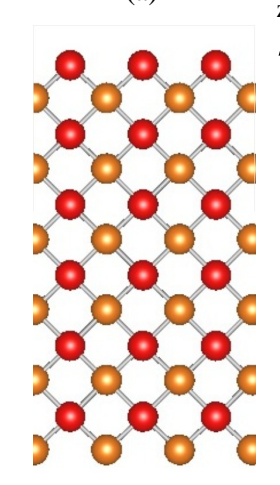

$\mathrm{z}$
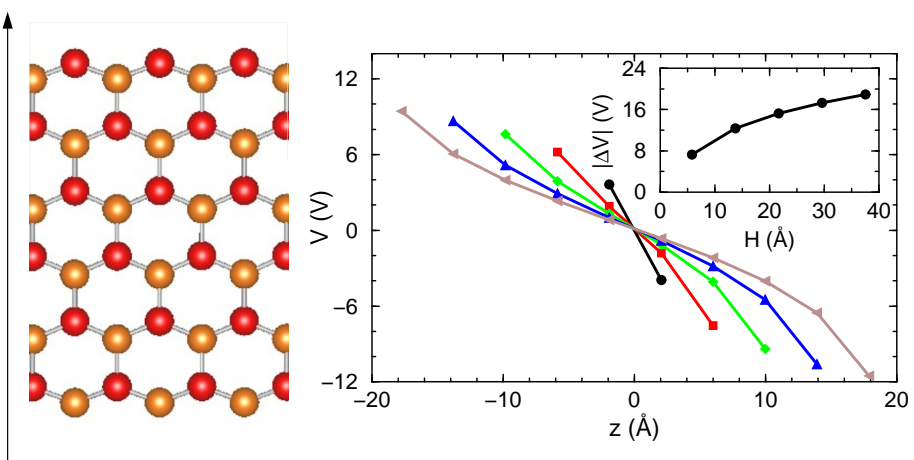

Fig. 11 Top view of polar nano-ribbons cut out of (a) a rock salt (001) ML with [110] edges or (b) an h-BN(0001) ML with ZZ edges. (c): Variations of the electrostatic potential across polar ribbons of increasing width $H=6,14,22,30$, and $38 \AA$. The inset highlights the logarithmic dependence of $|\Delta V|$ as a function of $H$.

\subsection{Unsupported polar ribbons}

Considering the similarities of the electrostatic properties between polar thin films and polar ribbons, it is not surprising that the range of possible behaviors displayed by the latter largely overlaps those described in Section 3. In the following, we will nevertheless highlight some distinctive features of polar ribbons.

In the electronic reconstruction mechanism, due to the increase of $\Delta V$ with $N$, eventually an overlap of the outermost row VB and CB takes place, yielding the compensating charges $\delta \lambda_{\text {comp }}$ and edge metallization(1DEG). The LDOS narrowing on edge atoms, due to their reduced coordination number, which is particularly strong on the anion terminations, favors the stabilization of spin polarization together with the edge metallization, as at thin film polar surfaces. $\Delta V$ does not vanish but stabilizes at a value of the order of the ribbon gap. This finite value constrains $\delta \lambda_{\text {comp }}$ to depart from $P_{M L}$ :

$$
\delta \lambda_{\text {comp }}(N) \neq P_{M L}
$$

and to vary with the ribbon width $N$ according to the following asymptotic law [90]:

$$
\delta \lambda_{\text {comp }}(N) \approx P_{M L}-\frac{\Delta V \varepsilon^{\infty}}{4 \ln N}
$$


This expression bears a close resemblance to the corresponding expression for thin films, Equation 5, except for the logarithmic function of size in the denominator which replaces the linear function in films.
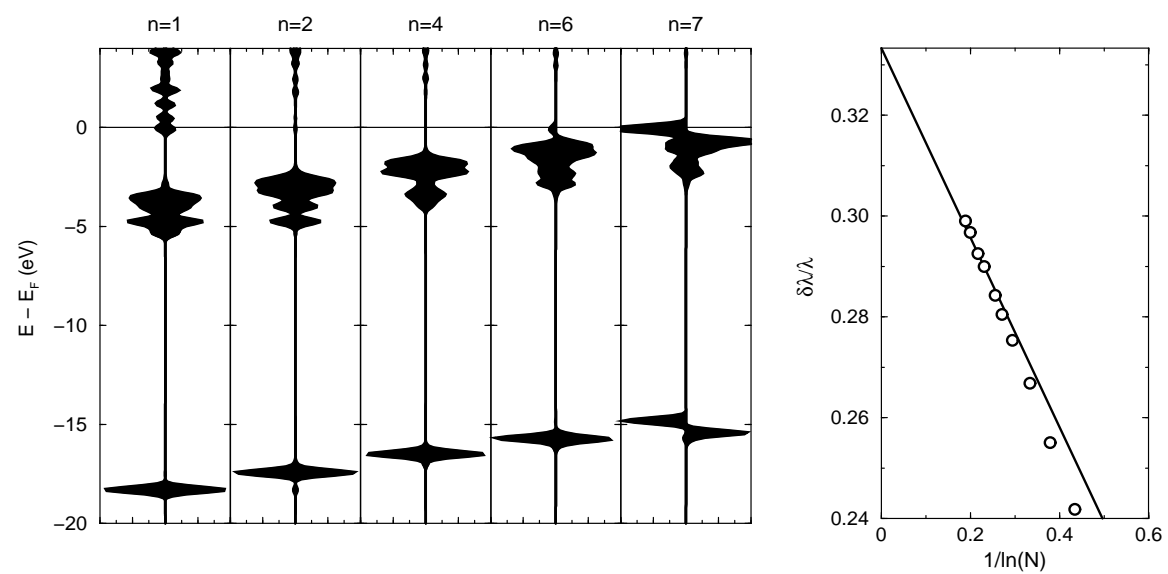

Fig. 12 Left: LDOS through an $\mathrm{MgO} \mathrm{ZZ}$ ribbon of width $N=7$, highlighting the metallization of the surfaces and the band shifts across the ribbon. Row $n=1$ is a cationic row. For simplicity, LDOS of rows $n=3$ and $n=5$ are not plotted. Right: Width dependence of $\delta \lambda / \lambda$ represented as a function of $1 / \ln N$ to highlight its asymptotic behavior.

Such scenario has been found in polar ribbons of various oxides, such as $\mathrm{MgO}$ [90, 91], $\mathrm{ZnO}[92,93,94,95], \mathrm{BeO}[41], \mathrm{V}_{2} \mathrm{O}_{5}$ [96], as well as in non-oxide compounds $\mathrm{MoS}_{2}$ [90, 97, 98, 99, 100, 101, 102, 103], ZnS [104], BN [105, 106, 107], $\mathrm{AlN}, \mathrm{GaN}$ and $\mathrm{SiC}[108,109]$. In most cases, the authors did not make reference to polarity. In $\mathrm{MgO} \mathrm{ZZ}$ ribbons, however, the LDOS and the size variation of the excess charges, displayed in Fig. 12, clearly exemplify the electronic reconstruction scenario and the validity of Equation 17 [90]. By a detailed comparison between $\mathrm{ZZ}$ ribbons of $\mathrm{MgO}$ and $\mathrm{MoS}_{2}$, it was also shown that polar signatures depend only marginally on the degree of ionicity of the compound [90]. It is important to stress that edge metallicity in these polar ribbons results from polarity compensation and is associated to an effective charge transfer between the two opposite edges. This is not the case in graphene $\mathrm{ZZ}$ nano ribbons, which are non-polar and in which states at the Fermi level are non-bonding $\pi$ states localized at low coordinated edge atoms, with no correlation to charge transfer processes across the ribbon.

Compensating charges may also be provided by non-stoichiometry in the outermost rows, or adsorption of charged species such as protons or hydroxyl groups issued from water dissociation. As in polar thin films, these mechanisms are generally more efficient than electronic reconstruction. In Fig. 13, the stability diagrams of $\mathrm{MgO}(111)$ polar surfaces $[110,111]$ and $\mathrm{MgO} \mathrm{ZZ}$ polar ribbons [91] highlight their similar behavior, despite the difference in dimensionality. 

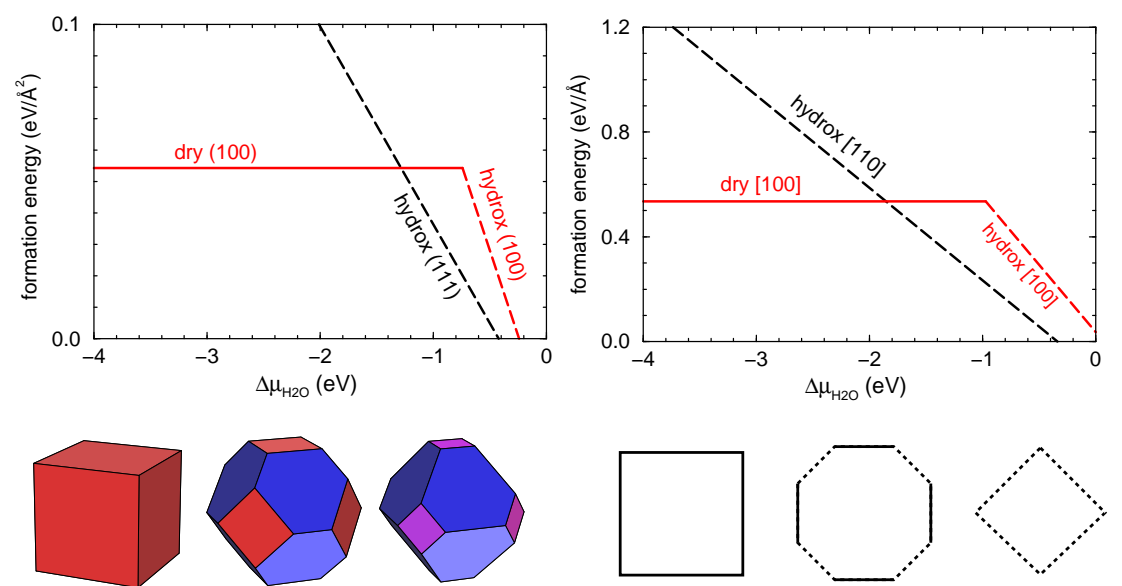

Fig. 13 Left: Formation energies $\left(\mathrm{eV} / \AA^{2}\right)$ of polar $\mathrm{MgO}(111)$ and non-polar $\mathrm{MgO}(001)$ surfaces as a function of water chemical potential $\mu_{\mathrm{H}_{2} \mathrm{O}}$. Right: Formation energies (eV/Å) of polar $\mathrm{MgO}$ [110] and non-polar $\mathrm{MgO}$ [100] ribbons, as a function of $\mu_{\mathrm{H}_{2} \mathrm{O}}$. Bottom panels display the corresponding Wulff shapes at low, medium and high water chemical potentials, from left to right. Dry (100) facets, hydroxylated (100) facets and hydroxylated (111) facets of 3D particles are represented in red, purple and blue, respectively. Dry [100] and hydroxylated [110] edges of 2D islands are represented by plain and dashed lines, respectively.

Edge energies of infinite ribbons enable the determination of large island shapes on the basis of the Wulff construction. Results for unsupported $\mathrm{MgO}$ islands (Figure 13) at increasing values of water chemical potential show a progressive transition from dry non-polar [100] to hydroxylated polar [110] edges [91]. This trend is the 2D equivalent of the transition from cubic shapes with dry (100) facets to octahedral shapes with hydroxylated (111) facets found in 3D MgO particles [110, 111].

Interestingly, according to simulations of $\mathrm{ZnO} \mathrm{ZZ}$ ribbons [92], the metallic character of their edges disappears at 2ML-thickness. Due to the h-BN(0001) stacking sequence in which oxygen atoms are located on-top cations and vice versa, there is a cancellation of polarity effects between the two layers, the potential difference induced by the top layer being equal and opposite to that of the bottom layer. No compensating charges are then needed and the ribbons keep their insulating or semiconducting character. This reasoning may be extended to other compounds displaying the h-BN structure and to thicker ribbons in which an odd-even alternation of the electronic structure takes place as a function of thickness. It has no equivalent in the case of polar thin films. 


\subsection{Finite size effects}

Polarity compensation cannot be avoided in large width polar ribbons. However, similarly to ultra-thin films, different scenarios may be expected at small width, in which charge compensation $\delta \lambda_{\text {comp }}$ may substantially differ from the actual polarization $P$ and/or $P$ may differ from $P_{M L}$.

A spontaneous transformation towards a structure with non-polar edges pertains to the case where $\delta \lambda_{\text {comp }}=P=0$ and thus $P \neq P_{M L}$. It may occur in small width ribbons due to their important edge-to-central atom ratio. The new structure being different from the ML ground state, there is a cost of energy for each additional 2D repeat unit when the width $N$ increases. However, as long as $N$ remains small, the gain in edge energy in having a non-polar orientation is able to overcome this cost. Thus, up to a critical width, ultra-thin polar ribbons may sustain a structure which is not their ML ground state but allows non-polar edges.

$1 \mathrm{~T}$

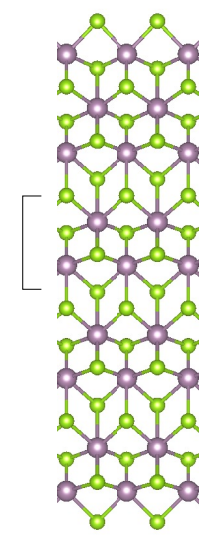

$1 \mathrm{H}$

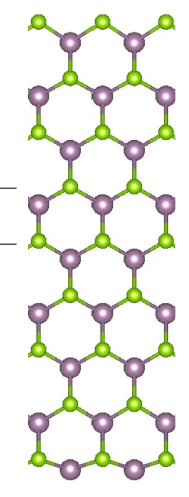

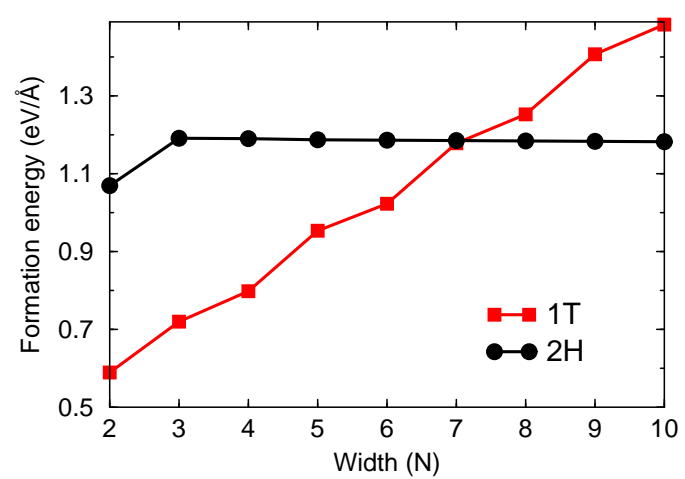

Fig. 14 Left panels: Top views of $1 \mathrm{~T}$ and $1 \mathrm{H} \mathrm{MoS} 2 \mathrm{ZZ}$ ribbons. Mo and $\mathrm{S}$ atoms are represented by large purple and small green balls, respectively. Note that the $1 \mathrm{~T}$ repeat units bears no dipole moment at variance with the $1 \mathrm{H}$ one. Right panel; formation energy of $1 \mathrm{~T}$ and $1 \mathrm{H} \mathrm{MoS} 2 \mathrm{ZZ}$ ribbons as a function of their width $N$.

This scenario has been invoked in $\mathrm{ZZ}$ ribbons of $\mathrm{MoS}_{2}, \mathrm{WS}_{2}$ and $\mathrm{MoSe}_{2}$ [112]. In the infinite trilayer, their structural ground state, labeled $1 \mathrm{H}$, is such that each TM has a trigonal prismatic coordination with the nearby S/Se atoms. Higher in energy, is a metastable $1 \mathrm{~T}$ phase, in which each TM is octahedrally coordinated to its neighbors. $\mathrm{ZZ}$ edges of $1 \mathrm{~T}$ ribbons are non-polar, while $\mathrm{ZZ}$ edges of $1 \mathrm{H}$ ribbons are polar (Figure 14). The 1T phase is thus stabilized in narrow ZZ ribbons up to a critical width $N_{c}$ of the order of a few repeat units. Such structural transformation does not take place in $\mathrm{ZrS}_{2} \mathrm{ZZ}$ ribbons since the ML ground state is already $1 \mathrm{~T}$. An interesting outcome of the $1 \mathrm{H}$-to- $1 \mathrm{~T}$ transformation, which is expected to drastically 
change the ribbon transport as well as optical properties, is the existence of a sound modification in their electronic properties at $N_{c}$, due to the different electronic character of the 1T and 1H MLs: metallic in the 1T phase and semiconducting in the $1 \mathrm{H}$ phase.

To our knowledge, there is presently no example of uncompensated polarity (in which $\delta \lambda_{\text {comp }}=0$ and $\delta \lambda_{\text {comp }} \neq P$ ), in polar nano-ribbons. The flexibility of these nano-structures, much stronger than thin films, may give hints for the absence of this regime. For example, in our study of $\mathrm{ZZ} \mathrm{MgO}$ ribbons [91], we found that below $N=6$, there is a spontaneous transformation of $\mathrm{ZZ}$ ribbons towards a non-polar [100] configuration, preventing the occurrence of uncompensated polarity.

\subsection{Interfaces between polar ribbons}

In line with the similarities of behaviors between polar films and polar ribbons, the interface characteristics between two polar ribbons are driven by the strength of the polarization discontinuity $\delta P$, which, in $2 \mathrm{D}$, reads:

$$
\delta P=P-P^{\prime}=\mathscr{R}\left(\Lambda-\Lambda^{\prime}\right)+\Lambda^{\prime}\left(\mathscr{R}-\mathscr{R}^{\prime}\right)+\delta P_{e l}
$$

In the absence of polarization discontinuity $(\delta P=0)$, the interface will remain insulating, while a finite discontinuity $(\delta P \neq 0)$ will lead either to electronic reconstruction at large width or to uncompensated polarity under a critical width.

Such polar/polar interfaces between 2D ribbons have been predicted to accommodate fully spin-polarized $1 \mathrm{DEG}$ or $1 \mathrm{DHG}$ in the case of $\mathrm{AlN} / \mathrm{SiC}$ and $\mathrm{ZnO} / \mathrm{SiC}$ $\mathrm{ZZ}$ ribbons [113]. Indeed these interfaces exemplify cases in which the polarization discontinuity is driven by the valence discontinuity with $\Lambda-\Lambda^{\prime}=1$ in the former and 2 in the latter, per unit cell length. Consistently, the interfacial compensating charge density is nearly twice larger in $\mathrm{ZnO} / \mathrm{SiC}$ than in $\mathrm{AlN} / \mathrm{SiC}$, the small discrepancy with respect to the ratio 2 being due to the electronic contribution to $\delta P$ in these non-centro-symmetric honeycomb lattices. Considering the fact that these compounds have not been isolated as ML, the authors of Ref. [114] have suggested several pathways to engineer polar discontinuities, involving selective functionalization of a BN ML or total functionalization of graphene/BN interfaces.

\subsection{Support effects}

The scenarios described previously apply to (unsupported) polar ribbons. Although interesting because they allow to point out the intrinsic effect of polarity, they can only represent a preliminary step towards a full understanding of the behavior of supported polar ribbons. 
Similarly to what happens at the interface between a polar thin film and its metallic support (Section 3.4), compensation of polarity in supported ribbons involves screening effects by the support. A large part of the compensating charges is transferred from the oxide edges to the metal support, thus allowing the oxide ions to recover charges closer to their ML values. In that way, the polar instability is healed and the interfacial electron transfer leads to strong adhesion along the ribbon edges. The efficiency of the metal screening is even stronger for polar ribbons than for polar films, since both ribbon edges interact with the support, while screening occurs at a single film termination, the one in contact with the support.
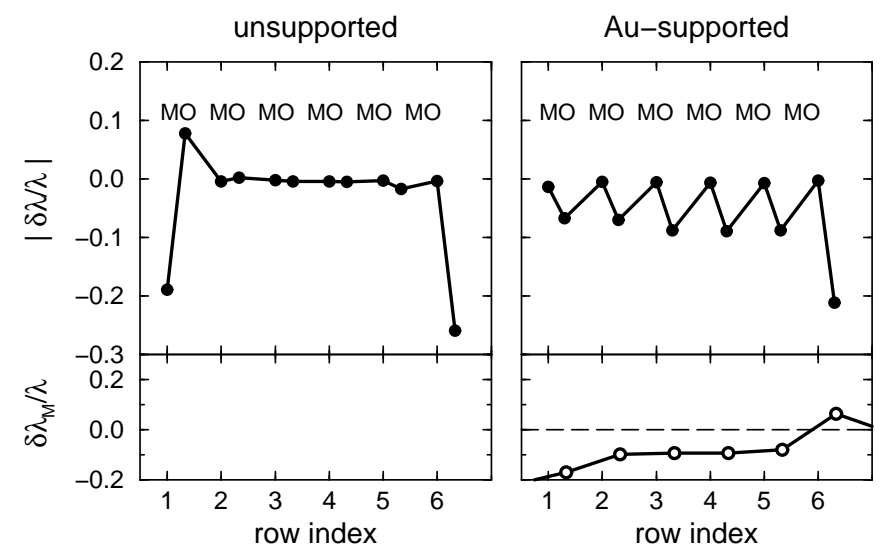

Fig. 15 Bader excess charge $\delta \lambda / \lambda$ at anion and cation sites across unsupported and Au-supported $\mathrm{MgO} Z \mathrm{ZZ}$ ribbons. Bottom panels represent the modification of charge $\delta \lambda_{M} / \lambda$ in the metal substrate underneath the ribbon. Note that screening at the $\mathrm{Mg}$ edge (row \#1) is more efficient than at the $\mathrm{O}$ edge, due to the large electronegativity of gold.

The few theoretical works which have considered metal-supported polar ribbons $[90,91,115,116]$ support this picture. For example, Fig. 15 compares the charge distribution in unsupported and $\mathrm{Au}$-supported $\mathrm{MgO} \mathrm{ZZ}$ ribbons [91]. It evidences an electron excess in the metal below the ribbon cation edge and an electron depletion under its oxygen edge.

The relative efficiency of electronic reconstruction, non-stoichiometry and hydroxylation in compensating polarity may be strongly influenced by the support. In particular, since the stabilization due to the screening of the compensating charges by the metal on both sides of supported polar ribbons is very efficient, electronic reconstruction becomes competitive with respect to non-stoichiometry and hydroxylation, allowing polar ribbons to remain dry and unreconstructed in a large range of water chemical potentials. These general features are exemplified in the case of $\mathrm{Au}$-supported $\mathrm{MgO} \mathrm{ZZ}$ ribbons (Figure 16) [91]. It turns out that, at variance with unsupported ribbons, supported dry and unreconstructed $\mathrm{ZZ}$ ribbons are more stable than AC ones. At intermediate water chemical potential, where differences between 
[100], [110], ZZ, and AC edge energies are tiny, islands of different symmetries may coexist. This is consistent with the recent experimental findings of shape transitions in $\mathrm{MgO}$ islands grown on the $\mathrm{Au}(111)$ surface $[117,118,119]$.
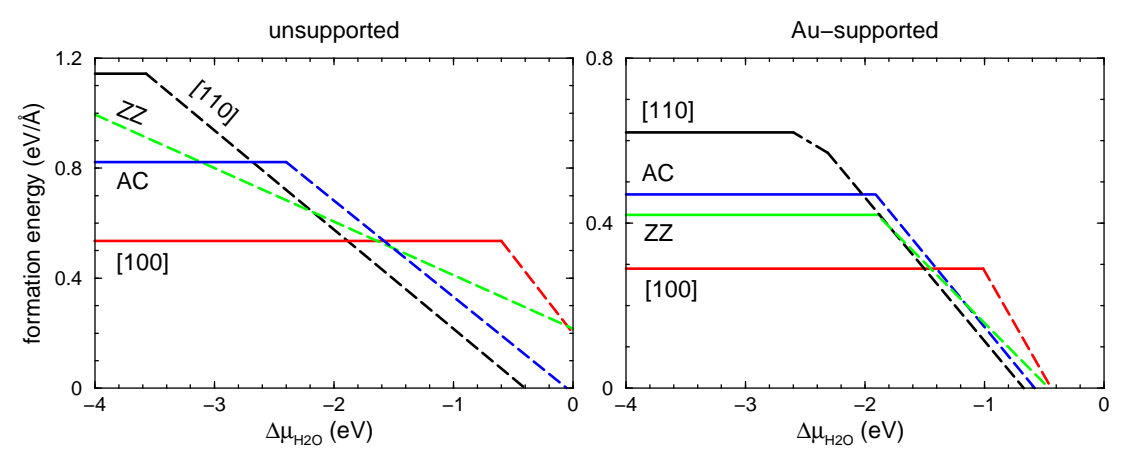

Fig. 16 Formation energies (eV/Å) of unsupported and Au-supported polar and non-polar $\mathrm{MgO}$ ribbons as a function of water chemical potential $\mu_{\mathrm{H}_{2} \mathrm{O}}$. Bare or fully hydroxylated states of the ribbons are represented with full and dashed lines, respectively [91].

To our knowledge, there are no experimental results on infinitely long polar ribbons. There are however hints of edge polarity in polar 2D islands in non-oxide compounds such as $\mathrm{MoS}_{2}[120,121]$, or BN [105, 106, 107, 122], although no relation to polarity was invoked. For example, STM experiments $[120,121]$ have evidenced the growth of $\mathrm{MoS}_{2}$ (0001) islands of triangular shape on $\mathrm{Au}(111)$, with edges displaying a pronounced contrast which was interpreted as due to the presence of metallic states [97, 123]. The observation of $\mathrm{MgO}$ and $\mathrm{NiO}$ mono- or bilayer islands with [110] polar edges, grown on $\operatorname{Ag}(001)[124,125,126]$ as well as simulations $[115,116]$ have suggested that these islands might be embedded in the substrate grooves. Let us note that an excess of oxygen in 2D islands stabilized under specific preparation conditions, such as observed in $\mathrm{MgO} / \mathrm{Ag}(100)[127,128]$ or $\mathrm{FeO}_{x} / \mathrm{Pt}(111)[65,66]$, may alter the relative stability of the various island edges.

This discussion proves that a metallic support may drastically change the properties of polar ribbons - atomic structure, electronic properties and stability compared to those expected when the ribbons are unsupported. In particular, in the electronic reconstruction scenario, the exciting electronic and magnetic properties of the 1DEG disappear, since the compensating charge, mainly localized in the support, becomes mixed with its metallic states.

\subsection{Polar nano-islands}

The previous analysis has mainly focused on (infinitely long) ribbons, but also applies to $2 \mathrm{D}$ large islands with polar edges, as long as their shape may be predicted 
from edge formation energies by the Wulff theorem. However, electrostatic considerations predict some specific finite size effects, driven by the finite length $L$ of the island edges.
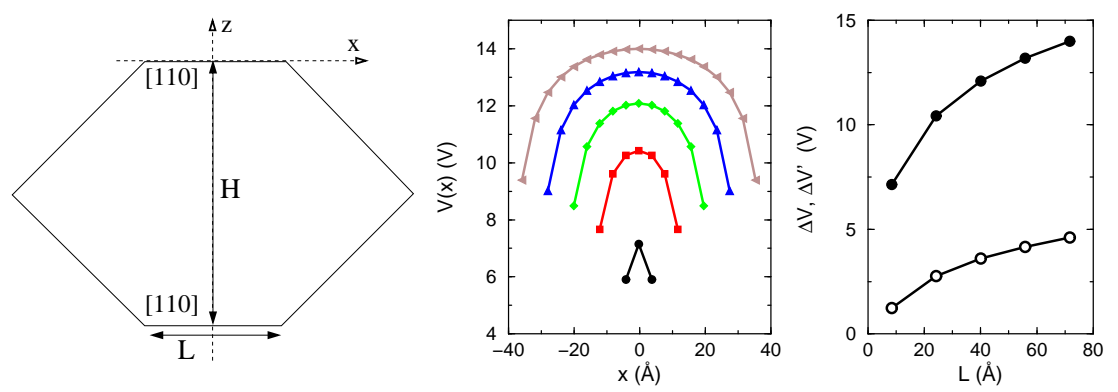

Fig. 17 Left panel: a 2D (001) island with two [110] polar edges. Middle panel: potential profile $V(x)$ along a polar edge in the limit $L \ll H$, for different values of $L(L=8 \AA, 34 \AA 40 \AA$, $55 \AA$, and $71 \AA$ ). Right panel: Potential difference $\Delta V$ between the center of the objects and the center of gravity of their polar termination (black symbols), and maximal potential variation $\Delta V^{\prime}$ within their polar terminations (open symbols).

Across an island, such as the one represented in Fig. 17, the voltage $\Delta V$ depends on both the island width $H$ and the edge length $L$ in the following way ( $a$ an atomic length) [89]:

$$
\Delta V=2 \delta \lambda \ln \left(\frac{L H}{a\left(L+\sqrt{L^{2}+4 H^{2}}\right)}\right)
$$

When $L \gg H$, the behavior of infinite ribbons is recovered, with $\Delta V$ increasing as $\ln H$. In the opposite limit $L \ll H$, relevant for nano-islands, $\Delta V$ grows as the logarithm of the lateral size $L$ of the polar termination:

$$
\Delta V=2 \delta \lambda \ln \left(\frac{L}{2 a}\right)
$$

It is thus the smallest of the two shape parameters, the width $H$ of the polar island or the length $L$ of its polar edge, which drives the electrostatic behavior. Ultimately, when the size of the polar edge is reduced to a single atom (1D wire), $\Delta V=2 \lambda \ln 2$ remains finite and independent of $H$.

Another finite size effect affects the potential profile along polar edges. In infinite ribbons, the average potential $V(x)$ along an edge is flat. This is no longer true in finite size islands in the limit $L \ll H$ (Figure 17). $V(x)$ presents large variations $\Delta V^{\prime}$ between the corners and the center of the edge, which scale as $\ln L$.

From these considerations, one could anticipate that polarity effects are less crucial in islands with small polar edges, and that the compensating charge density $\delta \lambda_{\text {comp }}$ in the regime $L \ll H$ might not be homogeneously distributed. As a consequence, vacancy formation energies might drastically depend on the edge length and on the location of the vacancy on the edge. Similarly, adsorption energies of charged 
species might depend on the precise adsorption site on the edge. However, these arguments remain speculative and need further confirmation whether by experiment or by simulation.

\section{Conclusion}

As reviewed in this chapter, electrostatic interactions and polarity effects are key driving forces behind many new compositions, structures and morphologies that oxides may display in the 2D limit. Most of the time, these new oxides have nothing to do with bulk oxides. While an intense activity has been devoted to polar thin films during the last decade, the synthesis of 2D polar nano-objects, such as ribbons or islands, is not as well-controlled and their properties not as well-understood. Several major challenges have to be taken up, such as defining preparation protocols, mastering the kinetics of the first steps of their formation, characterizing their composition and simulating their properties. The importance of the field has long been recognized in chemistry, due to the novel reactivity and selectivity properties displayed by these 2D oxides. Physical properties are nowadays also scrutinized, with a specific interest towards the consequences of confinement on the electronic degrees of freedom: edge/interface metallization, magnetism, and, in some cases, superconductivity. No doubt that a convergent effort of interdisciplinary nature will allow a deeper understanding and mastering of these objects in a near future.

\section{References}

1. Weiss W, Ranke W (2002) Surface chemistry and catalysis on well-defined epitaxial ironoxide layers. Prog Surf Sci 70: 1-151

2. Chen MS, Goodman DW (2008) Ultrathin, ordered oxide films on metal surfaces. J Phys : Condens Matter 20:264013

3. Freund H-J, Pacchioni G (2008) Oxide ultra-thin films on metals: new materials for the design of supported metal catalysts. Chem Soc Rev 37:2224-2242

4. Nilius N (2009) Properties of oxide thin films and their adsorption behavior studied by scanning tunneling microscopy and conductance spectroscopy. Surf Sci Rep 64:595-659

5. Wu Q-H, Fortunelli A, Granozzi G (2009) Preparation, characterisation and structure of Ti and Al ultrathin oxide films on metals. Int Rev Phys Chem 28:517-576

6. Netzer FP, Allegretti F, Surnev S (2010) Low-dimensional oxide nanostructures on metals: Hybrid systems with novel properties. J Vac Sci Technol B 28:1-16

7. Noguera C (2000) Polar oxide surfaces. J Phys Cond Matter 12:R367-R410

8. Goniakowski J, Finocchi F, Noguera C (2008) Polarity of oxide surfaces and nanostructures. Rep Prog Phys 71:016501

9. Tasker PWJ (1979) Stability of ionic crystal surfaces. J Phys C 12:4977-4984

10. King-Smith RD, Vanderbilt D (1993) Theory of polarization of crystalline solids. Phys Rev B 47:1651-1654

11. Stengel M, Vanderbilt D (2009) Berry-phase theory of polar discontinuities at oxide-oxide interfaces. Phys Rev B 80:241103 
12. Stengel M (2011) Electrostatic stability of insulating surfaces: Theory and applications. Phys Rev B 84:205432

13. Garcia A, Cohen ML (1993) 1st principles ionicity scales. 1 Charge asymmetry in the solidstate. Phys Rev B 47:4215-4221

14. Belabbes A, Furthmüller J, Bechstedt F (2013) Relation between spontaneous polarization and crystal field from first principles. Phys Rev B 87:0335305

15. Bristowe NC, Ghosez P, Littlewood PB, Artacho E (2014) The origin of two-dimensional electron gases at oxide interfaces: insights from theory. J Phys : Condens Matter 26:143201

16. Noguera C, Goniakowski J (2013) Polarity in Oxide Nano-objects. Chem Rev 113:4073-4105

17. Goniakowski J, Noguera C, Giordano L (2004) Using polarity for engineering oxide nanostructures: Structural phase diagram in free and supported $\mathrm{MgO}(111)$ ultrathin films. Phys Rev Lett 93:215702

18. Goniakowski J, Noguera C, Giordano L (2007) Prediction of uncompensated polarity in ultrathin films. Phys Rev Lett 98:205701

19. Gragnaniello L, Agnoli S, Parteder G, Barolo A, Bondino F, Allegretti F, Surnev S, Granozzi G, Netzer FP (2010) Cobalt oxide nanolayers on Pd(100): The thickness-dependent structural evolution. Surf Sci 604:2002-2011

20. Weirum G, Barcaro G, Fortunelli A, Weber F, Schennach R, Surnev S, Netzer FP (2010) Growth and Surface Structure of Zinc Oxide Layers on a Pd(111) Surface. J Phys Chem C 114:15432-15432

21. Schennach R, Weber F, Piffl M, Weirum G, Surnev S (2012) Growth and reactivity of Zn and $\mathrm{ZnO}$ on $\mathrm{Pd}(111)$. Surf Eng 28:87-90

22. Noguera C, Goniakowski J (2008) Polarity in oxide ultrathin films. J Phys : Condens Matter 20:264003

23. Kresse G, Dulub O, Diebold U (2003) Competing stabilization mechanism for the polar $\mathrm{ZnO}(0001)-\mathrm{Zn}$ surface. Phys Rev B 68:245409

24. Thiel S, Hammerl G, Schmehl A, Schneider CW, Mannhart J (2006) Tunable quasi-twodimensional electron gases in oxide heterostructures. Science 313:1942-1945

25. Hu XL, Michaelides A (2010) The kaolinite (001) polar basal plane. Surf Sci 604:111-117

26. Ohtomo A, Hwang HY (2004) A high-mobility electron gas at the $\mathrm{LaAlO}_{3} / \mathrm{SrTiO}_{3}$ heterointerface. Nature 427:423-426

27. Nakagawa N, Hwang HY, Muller DA (2006) Why some interfaces cannot be sharp. Nat Mater 5:204-209

28. Pentcheva R, Pickett WE (2006) Charge localization or itineracy at $\mathrm{LaAlO}_{3} / \mathrm{SrTiO}_{3}$ interfaces: Hole polarons, oxygen vacancies, and mobile electrons. Phys Rev B 74:035112

29. Reyren N, Thiel S, Caviglia AD, Kourkoutis LF, Hammerl G, Richter C, Schneider CW, Kopp T, Rüetschi A-S, Jaccard D, Gabay M, Muller DA, Triscone J-M, Mannhart J (2007) Superconducting interfaces between insulating oxides. Science 317:1196-1199

30. Gabay M, Gariglio S, Triscone JM, Santander-Syro AF (2013) 2-Dimensional oxide electronic gases: Interfaces and surfaces. Eur Phys J Special Topics 222:1177-1183

31. Segal Y, Ngai JH, Reiner JW, Walker FJ, Ahn CH (2009) X-ray photoemission studies of the metal-insulator transition in $\mathrm{LaAlO}_{3} / \mathrm{SrTiO}_{3}$ structures grown by molecular beam epitaxy. Phys Rev B 80:241107

32. Slooten E, Zhong Z, Molegraaf HJA, Eerkes PD, de Jong S, Massee F, van Heumen E, Kruize MK, Wenderich S, Kleibeuker JE, Gorgoi M, Hilgenkamp H, Brinkman A, Huijben M, Rijnders G, Blank DHA, Koster G, Kelly PJ, Golden MS (2013) Hard x-ray photoemission and density functional theory study of the internal electric field in $\mathrm{SrTiO}_{3} / \mathrm{LaAlO}_{3}$ oxide heterostructures. Phys Rev B 87:085128

33. Kiguchi M, Entani S, Saiki K, Goto T, Koma A (2003) Atomic and electronic structure of an unreconstructed polar $\mathrm{MgO}(111)$ thin film on $\mathrm{Ag}(111)$. Phys Rev B 68:115402

34. Ritter M, Ranke W, Weiss W (1998) Growth and structure of ultrathin FeO films on Pt(111) studied by STM and LEED. Phys Rev B 57:7240-7251

35. Ranke W, Ritter M, Weiss W (1999) Crystal structures and growth mechanism for ultrathin films of ionic compound materials: $\mathrm{FeO}(111)$ on $\mathrm{Pt}(111)$. Phys Rev B 60:1527-1530 
36. Gubo M, Ebensperger C, Meyer W, Hammer L, Heinz K (2009) Substoichiometric cobalt oxide monolayer on $\operatorname{Ir}(100)-(1$ x 1). J Phys : Condens Matter 21:474211

37. Ebensperger C, Gubo M, Meyer W, Hammer L, Heinz K (2010) Substrate-induced structural modulation of a $\mathrm{CoO}(111)$ bilayer on $\operatorname{Ir}(100)$. Phys Rev B 81:235405

38. Tusche C, Meyerheim HL, Kirschner J (2007) Observation of depolarized ZnO(0001) monolayers: Formation of unreconstructed planar sheets. Phys Rev Lett 99:026102

39. Claeyssens F, Freeman CL, Allan NL, Sun Y, Ashfold MN, Harding JH (2005) Growth of $\mathrm{ZnO}$ thin films - experiment and theory. J Mat Chem 15:139-148

40. Baumeier B, Krüger P, Pollmann J (2007) Structural, elastic, and electronic properties of SiC, $\mathrm{BN}$, and $\mathrm{BeO}$ nanotubes. Phys Rev B 76:085407

41. Wu W, Lu P, Zhang Z, Guo W (2011) Electronic and Magnetic Properties and Structural Stability of BeO Sheet and Nanoribbons. ACS Appl Mater Interfaces 3:4787-4795

42. Freeman CL, Claeyssens F, Allan NL, Harding JH (2006) Graphitic nanofilms as precursors to wurtzite films: Theory. Phys Rev Lett 96:066102

43. Kresse G, Schmid M, Napetschnig E, Shishkin M, Kohler L, Varga P (2005) Structure of the ultrathin aluminum oxide film on $\mathrm{NiAl}(110)$. Science 308:1440-1442

44. Goniakowski J, Noguera C (1999) Characteristics of Pd deposition on the $\mathrm{MgO}(111)$ surface. Phys Rev B 60:16120-128

45. Goniakowski J, Noguera C (2002) Microscopic mechanisms of stabilization of polar oxide surfaces: Transition metals on the $\mathrm{MgO}(111)$ surface. Phys Rev B 66:085417

46. Muller DA, Shashkov DA, Benedek R, Yang LH, Silcox J, Seidman DN (1998) Atomic scale observations of metal-induced gap states at $\{222\} \mathrm{MgO} / \mathrm{Cu}$ interfaces. Phys Rev Lett 80:47414744

47. Imhoff D, Laurent S, Colliex C, Backhaus-Ricoult M (1999) Determination of the characteristic interfacial electronic states of $\{111\} \mathrm{Cu}-\mathrm{MgO}$ interfaces by ELNES. Eur Phys J A 5:9-18

48. Tejedor C, Flores C, Louis E (1977) Metal-semiconductor interface- Si (111) and zincblende (110) junctions. J Phys C 10:2163-2177

49. Bordier G, Noguera C (1991) Electronic structure of a metal-insulator interface - Towards a theory of nonreactive adhesion. Phys Rev B 44:6361-6371

50. Goniakowski J, Noguera C (2004) Electronic states and Schottky barrier height at metal/MgO(100) interfaces. Interf Sci 12:93-103

51. Goniakowski J, Noguera C (2009) Polarization and rumpling in oxide monolayers deposited on metallic substrates. Phys Rev B 79:155433

52. Pacchioni G, Giordano L, Baistrocchi M (2005) Charging of metal atoms on ultrathin $\mathrm{MgO} / \mathrm{Mo}(100)$ films. Phys Rev Lett 94:226104

53. Giordano L, Pacchioni G (2006) Charge transfers at metal/oxide interfaces: a DFT study of formation of $\mathrm{K}^{+}$and $\mathrm{Au}^{-}$species on $\mathrm{MgO} / \mathrm{Ag}(100)$ ultra-thin films from deposition of neutral atoms. Phys Chem Chem Phys 8:3335-3341

54. Ricci D, Bongiorno A, Pacchioni G, Landman U (2006) Bonding trends and dimensionality crossover of gold nanoclusters on metal-supported MgO thin films. Phys Rev Lett 97:036106

55. Honkala K, Häkkinen $\mathrm{H}$ (2007) Au adsorption on regular and defected thin $\mathrm{MgO}$ (100) films supported by Mo. J Phys Chem C 111:4319-4327

56. Frondelius P, Hellman A, Honkala K, Häkkinen H, Grönbeck H (2008) Charging of atoms, clusters, and molecules on metal-supported oxides: A general and long-ranged phenomenon. Phys Rev B 78:085426

57. Sterrer M, Risse T, Pozzoni UM, Giordano L, Heyde M, Rust H-P, Pacchioni G, Freund H-J (2007) Control of the charge state of metal atoms on thin MgO films. Phys Rev Lett 98:096107

58. Sterrer M, Risse T, Heyde M, Rust H-P, Freund H-J (2007) Crossover from three-dimensional to two-dimensional geometries of $\mathrm{Au}$ nanostructures on thin $\mathrm{MgO}(001)$ films: A confirmation of theoretical predictions. Phys Rev Lett 98:206103

59. Barcaro G, Fortunelli A, Granozzi G (2008) Metal adsorption on oxide polar ultrathin films Phys Chem Chem Phys 10:1876-1882

60. Grönbeck H (2006) Mechanism for $\mathrm{NO}_{2}$ Charging on Metal Supported MgO. J Phys Chem B 110:11977-11981 
61. Hellman A, Klacar S, Grönbeck H (2009) Low Temperature CO Oxidation over Supported Ultrathin MgO Films. J Am Chem Soc 131:16636

62. Gonchar A, Risse T, Freund H-J, Giordano L, Di Valentin C, Pacchioni G (2011) Activation of Oxygen on $\mathrm{MgO}: \mathrm{O}_{2}^{-}$Radical Ion Formation on Thin, Metal-Supported $\mathrm{MgO}(001)$ Films. Angew Chem Int Ed 50:2635-2638

63. Goniakowski J, Noguera C, Giordano L, Pacchioni G (2009) Adsorption of metal adatoms on $\mathrm{FeO}(111)$ and $\mathrm{MgO}(111)$ monolayers: Effects of charge state of adsorbate on rumpling of supported oxide film. Phys Rev B 80:125403

64. Benedetti S, Stavale F, Valeri S, Noguera C, Freund H-J, Goniakowski J, Nilius N (2013) Steering the Growth of Metal Ad-particles via Interface Interactions Between a $\mathrm{MgO}$ Thin Film and a Mo Support. Adv Funct Materials 23:75-80

65. Sun YN, Qin ZH, Lewandowski M, Carrasco E, Sterrer M, Shaikhutdinov S, Freund H-J (2009) Monolayer iron oxide film on platinum promotes low temperature CO oxidation. J Catal 266:359-368

66. Sun YN, Giordano L, Goniaksowski J, Lewandowski M, Qin ZH, Noguera C, Shaikhutdinov S, Pacchioni G, Freund H-J (2010) The Interplay between Structure and CO Oxidation Catalysis on Metal-Supported Ultrathin Oxide Films. Angew Chem Int Ed 49:4418-4421

67. Giordano L, Lewandowski M, Groot IMN, Sun YN, Goniakowski J, Noguera C, Shaikhutdinov S, Pacchioni G, Freund H-J (2010) Oxygen-Induced Transformations of an $\mathrm{FeO}(111)$ Film on Pt(111): A Combined DFT and STM Study. J Phys Chem C 114:21504-21509

68. Giordano L, Pacchioni G, Noguera C, Goniakowski J (2014) Identification of Active Sites in a Realistic Model of Strong Metal-Support Interaction Catalysts: The Case of Platinum (111)-Supported Iron Oxide Film. ChemCatChem 6:185-190

69. He YB, Stierle A, Li WX, Farkas A, Kasper N, Over H (2008) Oxidation of $\operatorname{Ir}(111)$ : From O-Ir-O trilayer to bulk oxide formation. J Phys Chem C 112:11946-11953

70. Rogal J, Reuter K, Scheffler M (2008) CO oxidation on Pd(100) at technologically relevant pressure conditions: First-principles kinetic Monte Carlo study. Phys Rev B 77:155410

71. Gustafson J, Mikkelsen A, Borg M, Lundgren E, Köhler L, Kresse G, Schmid M, Varga P, Yuhara J, Torrelles X, Quirós C, Andersen JN (2004) Self-limited growth of a thin oxide layer on $\mathrm{Rh}(111)$. Phys Rev Lett 92:126102

72. Flege JI, Hrbek J, Sutter P (2008) Structural imaging of surface oxidation and oxidation catalysis on Ru(0001). Phys Rev B 78:165407

73. Franchini C, Podloucky R, Allegretti F, Li F, Parteder G, Surnev S, Netzer FP (2009) Structural and vibrational properties of two-dimensional $\mathrm{Mn}_{x} \mathrm{O}_{y}$ layers on $\mathrm{Pd}(100)$ : Experiments and density functional theory calculations. Phys Rev B 79:035420

74. Martynova Y, Soldemo M, Weissenrieder J, Sachert S, Polzin S, Widdra W, Shaikhutdinov S, Freund H-J (2013) CO Oxidation Over Monolayer Manganese Oxide Films on Pt(111). Catal Lett 143:1108-1115

75. Hwang HY, Iwasa Y, Kawasaki M, Keimer B, Nagaosa N, Tokura Y (2012) Emergent phenomena at oxide interfaces. Nature Mater 11:103-113

76. Mannhart J, Blank DHA, Hwang HY, Millis AJ, Triscone J-M (2008) Two-Dimensional Electron Gases at Oxide Interfaces. MRS Bulletin 333:1027-1034

77. Goniakowski J, Noguera C (2014) Conditions for electronic reconstruction at stoichiometric polar/polar interfaces. J Phys : Condens Matter 26:485010

78. Harrison A, Kraut EA, Waldrop JR and Grant RW (1978) Polar heterojunction interfaces. Phys Rev 18:4402-4410

79. Martin RM (1980) Atomic reconstruction at polar interfaces of semiconductors. J Vac Sci Technol 17:978-981

80. Tsukazaki A, Ohtomo A, Kita T, Ohno Y, Ohno H, Kawasaki M (2007) Quantum Hall effect in polar oxide heterostructures. Science:1388-1391

81. Das H, Spaldin NA, Waghmare UV, Saha-Dasgupta T (2010) Chemical control of polar behavior in bicomponent short-period superlattices. Phys Rev B 81:235112

82. Annadi A, Zhang Q, Wang XR, Tuzla N, Gopinadhan K, Lü WM, Barman AR, Liu ZQ, Srivastava A, Saha S, Zhao YL, Zeng SW, Dhar S, Olsson E, Gu B, Yunoki S, Maekawa S, Hilgenkamp H, Venkatesan T, Ariando (2013) Anisotropic two-dimensional electron gas at the $\mathrm{LaAlO}_{3} / \mathrm{SrTiO}_{3}$ (110) interface. Nature Comm 4:1838 
83. Herranz G, Sánchez F, Dix N, Scigaj M, Fontcuberta J (2012) High mobility conduction at (110) and (111) $\mathrm{LaAlO}_{3} / \mathrm{SrTiO}_{3}$ interfaces. Scientific Reports 2:758

84. Xue M, Guo Q, Wu K, Guo J (2009) Epitaxial growth of $\mathrm{ZnO}$ films on thin $\mathrm{FeO}(111)$ layers. J Cryst Growth 311:3918-3923

85. Betancourt J, Saavedra-Arias JJ, Burton JD, Ishikawa Y, Tsymbal EY, Velev JP (2013) Polarization discontinuity induced two-dimensional electron gas at $\mathrm{ZnO} / \mathrm{Zn}(\mathrm{Mg}) \mathrm{O}$ interfaces: A first-principles study. Phys Rev B 88:085418

86. Ohtomo A, Kawasaki M, Ohkubo I, Koinuma H, Yasuda T, Segawa Y (1999) Structure and optical properties of $\mathrm{ZnO} / \mathrm{Mg}_{0.2} \mathrm{Zn}_{0.8} \mathrm{O}$ superlattices. Appl Phys Lett 75:980-982

87. Dutta S, Pati SK (2010) Novel properties of graphene nanoribbons: a review. J Mater Chem 20:8207-8223

88. Acik M, Chabal YJ (2011) Nature of Graphene Edges: A Review. Jap J Appl Phys 50:070101

89. Goniakowski J, Noguera C (2011) Polarity at the nanoscale. Phys Rev B 83:115413

90. Güller F, Llois AM, Goniakowski J, Noguera C (2013) Polarity effects in unsupported polar nanoribbons. Phys Rev B 87:205423

91. Goniakowski J, Giordano L, Noguera C (2013) Polarity compensation in low-dimensional oxide nanostructures: The case of metal-supported MgO nanoribbons. Phys Rev B 87:035405

92. Botello-Mendez AR, Martinez-Martinez MT, Lopez-Urias F, Terrones M, Terrones H (2007) Metallic edges in zinc oxide nanoribbons. Chem Phys Lett 448:258-263

93. Botello-Mendez AR, Lopez-Urias F, Terrones M, Terrones H (2008) Magnetic behavior in zinc oxide zigzag nanoribbons. Nano Lett 8:1562-1565

94. Topsakal M, Cahangirov S, Bekaroglu E, Ciraci S (2009) First-principles study of zinc oxide honeycomb structures. Phys Rev B 80:235119

95. Wang Y, Wang B, Zhang Q, Shi D, Yunoki S, Kong F, Xu N (2012) A simple capacitor model and first-principles study of carbon-doped zigzag $\mathrm{ZnO}$ nanoribbons. Solid State Comm 152:534-539

96. Tang Q, Li F, Zhou Z, Chen Z (2011) Versatile Electronic and Magnetic Properties of Corrugated $\mathrm{V}_{2} \mathrm{O}_{5}$ Two-Dimensional Crystal and Its Derived One-Dimensional Nanoribbons: A Computational Exploration. J Phys Chem C 115:11983-11990

97. Bollinger MV, Lauritsen JV, Jacobsen KW, Nørskov JK, Helveg S, Besenbacher F (2001) One-dimensional metallic edge states in $\mathrm{MoS}_{2}$. Phys Rev Lett 87:196803

98. Li Y, Zhou Z, Zhang S, Chen Z (2008) $\mathrm{MoS}_{2}$ Nanoribbons: High Stability and Unusual Electronic and Magnetic Properties. J Am Chem Soc 130:16739-16744

99. Botello-Mendez AR, Lopez-Urias F, Terrones M, Terrones H (2009) Metallic and ferromagnetic edges in molybdenum disulfide nanoribbons. Nanotechnology 20:325703

100. Ataca C, Sahin H, Akturk E, Ciraci S (2011) Mechanical and Electronic Properties of $\mathrm{MoS}_{2}$ Nanoribbons and Their Defects. J Phys Chem C 115:3934-3941

101. Erdogan E, Popov IH, Enyashin AN, Seifert G (2012) Transport properties of $\mathrm{MoS}_{2}$ nanoribbons: edge priority. Eur Phys J B 85:33

102. Pan H, Zhang YW (2012) Edge-dependent structural, electronic and magnetic properties of $\mathrm{MoS}_{2}$ nanoribbons. J Mater Chem 22:7280-7290

103. Yang SQ, Li DX, Zhang TR, Tao ZL, Chen J (2012) First-Principles Study of Zigzag $\mathrm{MoS}_{2}$ Nanoribbon As a Promising Cathode Material for Rechargeable Mg Batteries. J Phys Chem C 116:1307-1312

104. Zhang S, Ma J (2011) Width- and Edge-Dependent Stability, Electronic Structures, and Magnetic Properties of Graphene-Like and Wurtzite ZnS Nanoribbons. J Phys Chem C 115:44664475

105. Ding Y, Wang Y, Ni J (2009) The stabilities of boron nitride nanoribbons with different hydrogen-terminated edges. Appl Phys Lett 94:233107

106. Chen W, Li Y, Yu G, Li C-Z, Zhang SB, Zhou Z, Chen Z, Chen J (2010) Hydrogenation: A Simple Approach To Realize Semiconductor-Half-Metal-Metal Transition in Boron Nitride Nanoribbons. J Am Chem Soc 132:1699-1705

107. Lopez-Bezanilla A, Huang J, Terrones H, Sumpter BG (2011) Boron Nitride Nanoribbons Become Metallic. Nano Lett 11:3267-3273 
108. Wu M, Wu X, Pei Y, Zeng XC (2011) Inorganic Nanoribbons with Unpassivated Zigzag Edges: Half Metallicity and Edge Reconstruction. Nano Res 4:233-239

109. Li H, Dai J, Li J, Zhang S, Zhou J, Zhang L, Chu W, Chen D, Zhao H, Yang J, Wu Z (2010) Electronic Structures and Magnetic Properties of GaN Sheets and Nanoribbons. J Phys Chem C 114:11390-11394

110. Finocchi F, Goniakowski J (2007) The effects of exchange and correlation on the computed equilibrium shapes of wet MgO crystallites. Surf Sci 601:4144-4148

111. Geysermans P, Finocchi F, Goniakowski J, Hacquart R, Jupille J (2009) Combination of (100), (110) and (111) facets in $\mathrm{MgO}$ crystals shapes from dry to wet environment. Phys Chem Chem Phys 11:2228-2233

112. Güller F, Llois AM, Goniakowski J, Noguera C (2015) Prediction of structural and metalto-semiconductor phase transitions in nanoscale $\mathrm{MoS}_{2}, \mathrm{WS}_{2}$, and other transition metal dichalcogenide zigzag ribbons. Phys Rev B 91:075407

113. Bristowe NC, Stengel M, Littlewood PB, Artacho E, Pruneda JM (2013) One-dimensional half-metallic interfaces of two-dimensional honeycomb insulators. Phys Rev B 88:161411

114. Gibertini M, Pizzi G, Marzari N (2014) Engineering polar discontinuities in honeycomb lattices. Nature Comm 5:5157

115. Ferrari AM, Casassa S, Pisani C (2005) Electronic structure and morphology of MgO submonolayers at the $\mathrm{Ag}(001)$ surface: An ab initio model study. Phys Rev B 71:155404

116. Ferrari AM, Casassa S, Pisani C, Altieri S, Rota A, Valeri S (2005) Polar and non-polar domain borders in $\mathrm{MgO}$ ultrathin films on $\mathrm{Ag}(001)$. Surf Sci 588:160-166

117. Benedetti S, Nilius N, Torelli P, Renaud G, Freund H-J, Valeri S (2011) Competition between Polar and Nonpolar Growth of MgO Thin Films on Au(111). J Phys Chem C 115:23043

118. Pan Y, Benedetti S, Noguera C, Giordano L, Goniakowski J, Nilius N (2012) Compensating Edge Polarity: A Means To Alter the Growth Orientation of $\mathrm{MgO}$ Nanostructures on $\mathrm{Au}(111)$. J Phys Chem C 116:11126

119. Nilius N, Benedetti S, Pan Y, Myrach P, Noguera C, Giordano L, Goniakowski J (2012) Electronic and electrostatic properties of polar oxide nanostructures: $\mathrm{MgO}(111)$ islands on $\mathrm{Au}(111)$. Phys Rev B 86:205410

120. Helveg S, Lauritsen JV, Lægsgaard E, Stensgaard I, Nørskov JK, Clausen BS, Topsøe H, Besenbacher F (2000) Atomic-scale structure of single-layer $\mathrm{MoS}_{2}$ nanoclusters. Phys Rev Lett 84:951-954

121. Lauritsen JV, Bollinger MV, Lægsgaard E, Jacobsen KW, Nørskov JK, Clausen BS, Topsøe H, Besenbacher F (2004) Atomic-scale insight into structure and morphology changes of $\mathrm{MoS}_{2}$ nanoclusters in hydrotreating catalysts. J Catal 221:510-522

122. Liu Y, Bhowmick S, Yakobson BI (2011) BN White Graphene with "Colorful" Edges: The Energies and Morphology. Nano Lett 11:3113-3116

123. Schweiger H, Raybaud P, Kresse G, Toulhoat H (2002) Shape and edge sites modifications of $\mathrm{MoS}_{2}$ catalytic nanoparticles induced by working conditions: A theoretical study. J Catal 207:76-87

124. Valeri S, Altieri S, del Pennino U, di Bona A, Luches P, Rota A (2002) Scanning tunnelling microscopy of $\mathrm{MgO}$ ultrathin films on $\mathrm{Ag}(001)$. Phys Rev B 65:245410

125. Caffio M, Atrei A, Cortigiani B, Rovida G (2006) STM study of the nanostructures prepared by deposition of $\mathrm{NiO}$ on $\mathrm{Ag}(001) \mathrm{J}$ Phys : Condens Matter 18:2379-2384

126. Steurer W, Allegretti F, Surnev S, Barcaro G, Sementa L, Negreiros F, Fortunelli A, Netzer FP (2011) Metamorphosis of ultrathin Ni oxide nanostructures on $\operatorname{Ag}(100)$. Phys Rev B $84: 115446$

127. Cabailh G, Lazzari R, Cruguel H, Jupille J, Savio L, Smerieri M, Orzelli A, Vattuone L, Rocca M (2011) Stoichiometry-Dependent Chemical Activity of Supported MgO(100) Films. J Phys Chem A 115:7161-7168

128. Pal J, Smerieri M, Celasco E, Savio L, Vattuone L, Ferrando R, Tosoni S, Giordano L, Pacchioni G, Rocca M (2014) How Growing Conditions and Interfacial Oxygen Affect the Final Morphology of MgO/Ag(100) Films. J Phys Chem C 118:26091-26102 


\section{Index}

1D electron/hole gas, 19, 23, 25

2D electron/hole gas, 7-9, 13, 15

Armchair edges, 18, 25

Charge density, 4, 18

compensating, 5-8, 11, 14, 17-20, 24, 26

excess, $4,14,18$

formal, 5, 6, 14, 18

interfacial transfer, 12, 24

Critical thickness, 9, 10, 16, 17

Critical width, 22, 23

Dipole moment, 4, 9, 12, 18

Finite size effects, 8-10, 16, 22, 26

Gap, 8, 11, 17, 19

Graphene, 18, 20, 23

Hydroxyl groups, 7, 20, 24

Interfaces, 11, 13-15, 24

Metal Induced Gap States, 12

Metal-insulator transition, 9, 16, 17, 23

Metallic supports, 4, 11, 24

Metallization, 7-9, 16, 19

Nano-islands, 18, 25

Nano-ribbons, 18

Point of zero charge, 12

Polar

catastrophe, 4

interfaces, 13-17, 23 orientation, $4,13,18$

ribbon/island edges, 18, 22, 25

ribbons, 18-20, 23, 24

surfaces, 4, 7, 19, 20

thin films, 7-11, 18, 21

Polarity, 4-6

compensation, 5, 12, 14, 20, 22, 24

dimensionality effects, $4,18,20$

stability criterion, 5, 7, 14, 18

uncompensated, 9, 12, 16, 23

Polarization, 5, 13, 14, 18, 22

covalent effects, 5,14

discontinuity, 7, 14, 16, 23

electronic, 5, 6, 14, 16

modern theory of, 5

spin polarization, 8,19

Polaronic distortion, 12

Reconstructions

electronic, 7, 8, 14, 15, 19, 23, 24

non-stoichiometric, 5, 14, 24

Schottky barrier height, 12

Subcritical regime, 9, 16

Surfaces, 4, 8, 10, 11, 14

Thin films, 7-13, 17

Transition metal dichalcogenides, 18, 20, 22, 25

Wannier function, 5

Wulff shapes, 21, 26

Zener breakdown, 7, 8

Zigzag edges, 18, 20-24 